\title{
Hydroselenation of Alkynes by Lithium Butylselenolate: An Approach in the Synthesis of Vinylic Selenides
}

\author{
Gilson Zeni, ${ }^{\text {a,* }}$ Marcelo P. Stracke, ${ }^{a}$ Cristina W. Nogueira, ${ }^{a}$ \\ Antonio L. Braga, ${ }^{a}$ Paulo H. Menezes, ${ }^{b}$ Helio A. Stefani ${ }^{c}$ \\ ${ }^{a}$ Laboratório de Síntese, Reatividade, Avaliação, Toxicológica e Farmacológica de \\ Organocalcogenios-CCNE-UFSM - 97105-900-Santa Maria - RS - Brazi l - \\ gzeni@quimica.ufsm.br \\ ${ }^{b}$ Departamento de Química Fundamental - UFPE - Recife - Brazil \\ ${ }^{\mathrm{c}}$ Faculdade de Ciências Farmacêuticas - USP - Sao Paulo - Brazil
}

\section{SUPPORTING INFORMATION}

\section{Materials and Methods}

Proton nuclear magnetic resonance spectra $\left({ }^{1} \mathrm{H}\right.$ NMR) were obtained at $200 \mathrm{MHz}$ on a Bruker DPX-200 NMR spectrometer or at $400 \mathrm{MHz}$ on a Bruker DPX-400 NMR spectrometer. Spectra were recorded in $\mathrm{CDCl}_{3}$ solutions. Chemical shifts are reported in ppm, referenced to the solvent peak of $\mathrm{CDCL}_{3}$ or tetramethylsilane (TMS) as the external reference. Data are reported as follows: chemical shift $(\delta)$, multiplicity, coupling constant $(J)$ in Hertz and integrated intensity. Carbon-13 nuclear magnetic resonance spectra $\left({ }^{13} \mathrm{C}\right.$ NMR) were obtained either at $50 \mathrm{MHz}$ on a Bruker DPX-200 NMR spectrometer or at 100 $\mathrm{MHz}$ on a Bruker DPX-400 NMR spectrometer. Spectra were recorded in $\mathrm{CDCl}_{3}$ solutions. Chemical shifts are reported in ppm, referenced to the solvent peak of $\mathrm{CDCL}_{3}$. Abbreviations to denote the multiplicity of a particular signal are s (singlet), $d$ (doublet), $t$ (triplet), q (quartet), quint (quintet), sex (sextet) and $\mathrm{m}$ (multiplet). High resolution mass spectra were recorded on a Kratos MS50TC double focusing magnetic sector mass spectrometer using EI at $70 \mathrm{eV}$. Column chromatography was performed using Merck Silica Gel (230-400 mesh) following the methods described by Still ${ }^{1}$. Thin layer chromatography (TLC) was performed using Merck Silica Gel GF $254,0.25$ mm thickness. For visualization, TLC plates were either placed under ultraviolet light, or stained with iodine vapor, or acidic vanillin. Most reactions were monitored by TLC for disappearance of starting material. The following solvents were dried and purified by distillation from the reagents indicated: tetrahydrofuran from sodium with a benzophenone ketyl indicator. All other solvents were ACS or HPLC grade unless otherwise noted. Air- and moisture-

\footnotetext{
${ }^{1}$ Still, W.C.; Kahn, M.; Mitra, A. J. Org. Chem. 1975, 43, 2923-2925
} 
sensitive reactions were conducted in flame-dried or oven dried glassware equipped with tightly fitted rubber septa and under a positive atmosphere of dry nitrogen or argon. Reagents and solvents were handled using standard syringe techniques. Temperatures above room temperature were maintained by use of a mineral oil bath with an electrically heated coil connected to a Variac controller.

Typical procedure for hydroselenation of alkynes by [BuSeLi]: To a suspension of the elemental selenium $(0.079 \mathrm{~g} ; 1 \mathrm{mmol})$ in dry THF $(5 \mathrm{~mL})$ under argon and magnetic stirring was added $n$-butyllithium $(0.4 \mathrm{~mL}$ of a $2.5 \mathrm{M}$ solution in hexane, $1 \mathrm{mmol})$. A yellow solution was formed. To this solution was added the appropriate alkynes ( $1 \mathrm{mmol})$ in ethanol $(10 \mathrm{~mL})$. The mixture was then heated at reflux for $24 \mathrm{~h}$. After this time, the mixture was cooled to room temperature and diluted with ethyl acetate $(20 \mathrm{~mL})$ and washed with brine $(2 \times 20 \mathrm{~mL})$. The organic phase was separated, dried over $\mathrm{MgSO}_{4}$ and concentrated under vacuum. The residue was purified by flash chromatography and eluted with hexane/ethyl acetate (80:20). Selected spectral and analytical data for 2a - (1Butylselanyl-2-phenyl-vinyl)-benzene: Yield: $0.189 \mathrm{~g}(60 \%) .{ }^{1} \mathrm{H} \mathrm{RMN}: \mathrm{CDCl}_{3}, 400 \mathrm{MHz}$, $\delta(\mathrm{ppm}): 7.50-7.17(\mathrm{~m}, 11 \mathrm{H}), 2.81(\mathrm{t}, J=7.3 \mathrm{~Hz}, 2 \mathrm{H}), 1.53$ (quint, $J=7.3 \mathrm{~Hz}, 2 \mathrm{H}), 1.34$ $(\mathrm{sex}, J=7.3 \mathrm{~Hz}, 2 \mathrm{H}), 0.87(\mathrm{t}, J=7.2 \mathrm{~Hz}, 3 \mathrm{H}) .{ }^{13} \mathrm{C} \mathrm{RMN}: \mathrm{CDCl}_{3}, 100 \mathrm{MHz}, \delta(\mathrm{ppm})$ : 137.85, 134.44, 131.28, 128.01, 123.02, 32.74, 29.76, 22.30, 13.29. IR (KBr, film) v 3028, 2892, 1702, 1597, 1492, 1210, 954. MS (EI, $70 \mathrm{eV}) \mathrm{m} / \mathrm{z}$ (relative intensity): 258 (12), 180 (100), 102 (77), 77 (45), 57 (21). HRMS Calcd. For $\mathrm{C}_{18} \mathrm{H}_{20} \mathrm{Se}$ : 316.07221. Found: 316.07148 .

4-Butylselanyl-3,8-dimethyl-dec-4-en-6-yne-3,8-diol - 2b: Yield: 0.182 g (55\%). ${ }^{1} \mathrm{H}$ RMN: $\mathrm{CDCl}_{3}, 400 \mathrm{MHz}, \delta(\mathrm{ppm}): 6.33(\mathrm{~s}, 1 \mathrm{H}), 3.04(\mathrm{t}, J=7.3 \mathrm{~Hz}, 2 \mathrm{H}), 2.23$ (s, 2H), 1.73$1.69(\mathrm{~m}, 4 \mathrm{H}), 1.52-1.38(\mathrm{~m}, 8 \mathrm{H}), 1.09-1.01(\mathrm{~m}, 5 \mathrm{H}), 0.93(\mathrm{t}, J=7.3 \mathrm{~Hz}, 3 \mathrm{H}), 0.86(\mathrm{t}, J=$ $7.3 \mathrm{~Hz}, 3 \mathrm{H}) .{ }^{13} \mathrm{C} \mathrm{RMN}: \mathrm{CDCl}_{3}, 100 \mathrm{MHz}, \delta(\mathrm{ppm}): 153.34,113.44,99.60,82.86,81.40$, $69.22,67.42,36.46,34.00,32.43,29.09,28.33,26.52,22.98,13.57,9.01,8.24$. IR ( $\mathrm{KBr}$, film) $\vee$ 3356, 3029, 2875, 1661, 1492, 1210, 957. MS (EI, $70 \mathrm{eV}) \mathrm{m} / z$ (relative intensity): 314 (44), 296 (17), 275 (23), 196 (100), 178 (54), 160 (33). HRMS Calcd. for $\mathrm{C}_{16} \mathrm{H}_{28} \mathrm{O}_{2} \mathrm{~S}$ : 332.13251. Found: 332.13322.

2-Butylselanyl-hex-2-en-4-yne-1,6-diol - 2c: Yield: 0.170 g (69\%). ${ }^{1} \mathrm{H}$ RMN: $\mathrm{CDCl}_{3}, 200$ $\mathrm{MHz}, \delta(\mathrm{ppm}): 6.16(\mathrm{t}, J=1.7 \mathrm{~Hz}, 1 \mathrm{H}), 4.43$ (s, 2H), 4.28 ( s, 2H), 2.97-2.87 (m, 4H), 1.74 (quint, $J=7.2 \mathrm{~Hz}, 2 \mathrm{H}), 1.51(\mathrm{sex}, J=7.2 \mathrm{~Hz}, 2 \mathrm{H}), 0.96(\mathrm{t}, J=7.2 \mathrm{~Hz}, 3 \mathrm{H}) .{ }^{13} \mathrm{C} \mathrm{RMN}$ : $\mathrm{CDCl}_{3}, 100 \mathrm{MHz}, \delta(\mathrm{ppm}):$ 146.80, 109,04, 94.55, 82.80, 66.01, 51.45, 32.67, 25.58, 22.85, 13.50. IR (KBr, film) v 3380, 3058, 2873, 1595, 1492, 1218, 954. MS (EI, $70 \mathrm{eV}) \mathrm{m} / z$ (relative intensity): 230( 40), 212(15), 191 (34), 112 (100), 94 (22), 76 (31). HRMS Calcd. for $\mathrm{C}_{10} \mathrm{H}_{16} \mathrm{O}_{2} \mathrm{Se}: 248.03653$. Found: 248.03781 .

(2-Butylselanyl-hex-1-enyl)-benzene - 2e: Yield: $0.141 \mathrm{~g}(48 \%) .{ }^{1} \mathrm{H} \mathrm{RMN}: \mathrm{CDCl}_{3}, 400$ MHz, $\delta(\mathrm{ppm}): 7.38-7.17(\mathrm{~m}, 5 \mathrm{H}), 6.89(\mathrm{~d}, J=2.3 \mathrm{~Hz}, 1 \mathrm{H}), 2.90(\mathrm{t}, J=7.3 \mathrm{~Hz}, 2 \mathrm{H}), 2.39(\mathrm{t}$, $J=7.2 \mathrm{~Hz}, 2 \mathrm{H}), 1.58-1.40(\mathrm{~m}, 8 \mathrm{H}), 0.88(\mathrm{t}, J=7.3 \mathrm{~Hz}, 3 \mathrm{H}) .{ }^{13} \mathrm{C} \mathrm{RMN}: \mathrm{CDCl}_{3}, 100 \mathrm{MHz}$, $\delta(\mathrm{ppm}): 134.01,128.74,128.39,128.18,128.13,128.01,127.27,124.09,32.98,30.78$, 29.74, 22.90, 22.53, 18.99, 13.67, 13.41. ). IR (KBr, film) v 3054, 2933, 1642, 1447, 1265, 
935. MS (EI, $70 \mathrm{eV}$ ) m/z (relative intensity): 239 (21), 160 (100), 83 (38), 77 (45). HRMS Calcd. for $\mathrm{C}_{16} \mathrm{H}_{24} \mathrm{Se}: 296.10582$. Found: 296.10741 .

2-Butylselanyl-3-phenyl-prop-2-en-1-ol - 2f: Yield: $0.175 \mathrm{~g}(65 \%) .{ }^{1} \mathrm{H}$ RMN: $\mathrm{CDCl}_{3}$, $200 \mathrm{MHz}, \delta(\mathrm{ppm}): 7.53-7.23(\mathrm{~m}, 5 \mathrm{H}), 7.03(\mathrm{~s}, 1 \mathrm{H}), 4.47(\mathrm{~s}, 2 \mathrm{H}), 2.77(\mathrm{t}, J=7.2 \mathrm{~Hz}, 2 \mathrm{H})$, $2.23(\mathrm{~s}, 1 \mathrm{H}), 1.65$ (quint, $J=7.3 \mathrm{~Hz}, 2 \mathrm{H}), 1.42-1.27(\mathrm{~m}, 2 \mathrm{H}), 0.81(\mathrm{t}, J=7.3 \mathrm{~Hz}, 3 \mathrm{H}) .{ }^{13} \mathrm{C}$ RMN: $\mathrm{CDCl}_{3}, 50 \mathrm{MHz}, \delta(\mathrm{ppm}): 136.73,132.97,131.14,131.60,129.03,128.23,127.95$, 127.30, 67.96, 32.35, 25.20, 23.81, 13.46. IR (KBr, film) v 3389, 2925, 2853, 1738, 1487, 1442, 952. MS (EI, $70 \mathrm{eV}$ ) $\mathrm{m} / z$ (relative intensity): 252 (40), 213 (23), 134 (100), 116 (10). HRMS Calcd. for $\mathrm{C}_{13} \mathrm{H}_{18}$ OSe: 270.05139 . Found: 270.05211.

(1-Butylselanyl-oct-1-en-3-ynyl)-benzene - 2g: Yield: $0.210 \mathrm{~g}(66 \%) .{ }^{1} \mathrm{H}$ RMN: $\mathrm{CDCl}_{3}$, $400 \mathrm{MHz}, \delta(\mathrm{ppm}): 7.5-7.2(\mathrm{~m}, 5 \mathrm{H}), 6.91(\mathrm{~s}, 1 \mathrm{H}), 2.89(\mathrm{t}, J=7.3,2 \mathrm{H}), 2.38(\mathrm{t}, J=7.3 \mathrm{~Hz}$, $2 \mathrm{H}), 1.68$ (quint, $J=7.3 \mathrm{~Hz}, 2 \mathrm{H}), 1.56-1.42(\mathrm{~m}, 6 \mathrm{H}), 0.96(\mathrm{t}, J=7.3 \mathrm{~Hz}, 6 \mathrm{H}) .{ }^{13} \mathrm{C}$ RMN: $\mathrm{CDCl}_{3}, 100 \mathrm{MHz}, \delta(\mathrm{ppm}): 136.77,132.48,128.92,128.31,127.30,126.21,124.71,122.16$, $84.82,65.05,34,60,32.58,31.45,22.64,21.95,19.27,13.82,13.51$. IR (KBr, film) v 3055, 2957, 2863, 1631, 1454, 959. MS (EI, $70 \mathrm{eV}$ ) $\mathrm{m} / \mathrm{z}$ (relative intensity): 263 (35), 184 (100), 107 (75), 77 (41). HRMS Calcd. for $\mathrm{C}_{18} \mathrm{H}_{24} \mathrm{Se}$ : 320.10557 . Found: 320.10682.

(1-Butylselanyl-4-phenyl-but-1-en-3-ynyl)-benzene - 2h: Yield: 0.227 g $(67 \%) .{ }^{1} \mathrm{H}$ RMN: $\mathrm{CDCl}_{3}, 200 \mathrm{MHz}, \delta(\mathrm{ppm}): 7.54-7.29(\mathrm{~m}, 10 \mathrm{H}), 6.26(\mathrm{~s}, 1 \mathrm{H}), 2.64(\mathrm{t}, J=6.7 \mathrm{~Hz}$, $2 \mathrm{H}$ ), 1.61 (quint, $J=7.2 \mathrm{~Hz}, 2 \mathrm{H}$ ), 1.37 (sex, $J=7.2 \mathrm{~Hz}, 2 \mathrm{H}$ ), $0.79\left(\mathrm{t}, J=7.2 \mathrm{~Hz}, 3 \mathrm{H}\right.$ ). ${ }^{13} \mathrm{C}$ RMN: $\mathrm{CDCl}_{3}, 100 \mathrm{MHz}, \delta(\mathrm{ppm}): 147.77,139.89,136.10,131.24,128.05,127.41,126.09$, 125.83, 123.44, 107.93, 96.70, 88.40, 32.36, 26.41, 22.77, 13.35. IR (KBr, film) v 3020, 2870, 2212, 1631, 1459, 1199, 960. MS (EI, $70 \mathrm{eV}$ ) m/z (relative intensity): 283 (28), 204 (100), 127 (29), 77 (40). HRMS Calcd. for $\mathrm{C}_{20} \mathrm{H}_{20} \mathrm{Se}: 340.07119$. Found: 340.07233.

3-Butylselanyl-2-methyl-6-phenyl-hex-3-en-5-yn-2-ol - 2i: Yield: 0.202 g $(63 \%) .{ }^{1} \mathrm{H}$ RMN: $\mathrm{CDCl}_{3}, 400 \mathrm{MHz}, \delta(\mathrm{ppm}): 7.46-7.28(\mathrm{~m}, 5 \mathrm{H}), 6.56(\mathrm{~s}, 1 \mathrm{H}), 3.13(\mathrm{t}, J=7.3 \mathrm{~Hz}, 2 \mathrm{H})$, $2.5(\mathrm{~s}, 1 \mathrm{H}), 1.69$ (quint, $J=7.3 \mathrm{~Hz}, 2 \mathrm{H}), 1.46-1.44(\mathrm{~m}, 8 \mathrm{H}), 0.87(\mathrm{t}, J=7.3 \mathrm{~Hz}, 3 \mathrm{H}) .{ }^{13} \mathrm{C}$ RMN: $\mathrm{CDCl}_{3}, 100 \mathrm{MHz}, \delta(\mathrm{ppm}): 155.44,131.12,128.98,128.64,128.18,128.14,126.87$, 123.27, 112.26, 96.04, 87.63, 74.58, 32.26, 29.04, 28.33, 22.80, 13.40. IR (KBr, film) v 3330, 2964, 2871, 1721, 1460, 1266, 958. MS (EI, $70 \mathrm{eV}$ ) $\mathrm{m} / \mathrm{z}$ (relative intensity): 304 (21), 265 (44), 186 (100), 168 (75), 77 (11). HRMS Calcd. for $\mathrm{C}_{17} \mathrm{H}_{22} \mathrm{OSe}$ : 322.085818. Found: 322.087731 .

1-Butylselanyl-1-methylsulfanyl-hex-1-ene - 2j: Yield: $0.168 \mathrm{~g}(59 \%) .{ }^{1} \mathrm{H}$ RMN: $\mathrm{CDCl}_{3}$, $400 \mathrm{MHz}, \delta(\mathrm{ppm}): 7.53-7.24(\mathrm{~m}, 5 \mathrm{H}), 6.20(\mathrm{~s}, 1 \mathrm{H}), 3.1(\mathrm{t}, J=7.2 \mathrm{~Hz}, 2 \mathrm{H}), 2.39-2.31(\mathrm{~m}$, $3 \mathrm{H}), 1.71-1.39(\mathrm{~m}, 4 \mathrm{H}), 0.94(\mathrm{t}, J=7.3 \mathrm{~Hz}, 3 \mathrm{H}) .{ }^{13} \mathrm{C} \mathrm{RMN}: \mathrm{CDCl}_{3}, 100 \mathrm{MHz}, \delta(\mathrm{ppm})$ : 133.15, 129.03, 128.51, 128.39, 128.23, 127.95, 127.30, 123.19, 33.69, 29.69, 23.02, 17.41, 13.57. IR (KBr, film) v 2959, 2864, 1607, 1455, 1237, 954. MS (EI, $70 \mathrm{eV}) \mathrm{m} / z$ (relative intensity): 229 (17), 150 (100), 103 (75). HRMS Calcd. for $\mathrm{C}_{13} \mathrm{H}_{18} \mathrm{SSe}: 286.03547$. Found: 286.03692 . 
(2-Butylselanyl-hex-1-enyl)-phosphonic acid diethyl ester - 2l: Yield: $0.227 \mathrm{~g}(64 \%) .{ }^{1} \mathrm{H}$ RMN: $\mathrm{CDCl}_{3}, 200 \mathrm{MHz}, \delta(\mathrm{ppm}): 5.93(\mathrm{~d}, J=13.6 \mathrm{~Hz}, 1 \mathrm{H}), 5.46(\mathrm{~d}, J=13.6 \mathrm{~Hz}, 1 \mathrm{H}), 4.14$ $\left(\mathrm{dq},{ }^{3} J_{\mathrm{PH}}=7.4 \mathrm{~Hz}, J=7.2 \mathrm{~Hz}, 4 \mathrm{H}\right), 3.02(\mathrm{t}, J=7.3 \mathrm{~Hz}, 4 \mathrm{H}), 1.73-1.25(\mathrm{~m}, 14 \mathrm{H}), 0.96-0.92$ $(\mathrm{m}, 6 \mathrm{H}) .{ }^{13} \mathrm{C} \mathrm{RMN}: \mathrm{CDCl}_{3}, 100 \mathrm{MHz}, \delta(\mathrm{ppm}): 164.45\left(\mathrm{~d},{ }_{P-C}=6.3 \mathrm{~Hz}\right), 139.35,114.25(\mathrm{~d}$, $\left.{ }^{1} J_{P-C}=188 \mathrm{~Hz}\right), 62.04\left(\mathrm{~d},{ }^{2} J_{P-C}=5 \mathrm{~Hz}\right), 36.37,32.46,30.65,25.92,23.12,22.93,22.29$, 21.92, $16.38\left(\mathrm{~d},{ }^{3} J_{P-C}=6 \mathrm{~Hz}\right), 13.81,13.486 . \mathrm{IR}(\mathrm{KBr}$, film $) \vee 3024,2861,1596,1488$, 1254, 1133, 1010, 955. MS (EI, $70 \mathrm{eV}$ ) $\mathrm{m} / z$ (relative intensity): 299 (23), 220 (100), 130 (31), 84 (17). HRMS Calcd. for $\mathrm{C}_{14} \mathrm{H}_{29} \mathrm{O}_{3}$ PSe: 356.10677. Found: 356.10812.

(2-Butylselanyl-vinyl)-benzene - 5a: Yield $0.191 \mathrm{~g}(80 \%) ;{ }^{1} \mathrm{H} \mathrm{RMN}: \mathrm{CDCl}_{3}, 200 \mathrm{MHz}$, $\delta(\mathrm{ppm}): 7.42-7.17(\mathrm{~m}, 5 \mathrm{H}) ; 6.89(\mathrm{~d}, J=10.6 \mathrm{~Hz} ; 1 \mathrm{H}) ; 6.62(\mathrm{~d}, J=10.6 \mathrm{~Hz} ; 1 \mathrm{H}) ; 2.81(\mathrm{t}, J$ $=7.3 \mathrm{~Hz}, 2 \mathrm{H}) ; 1.81$ (quint, $J=7.3 \mathrm{~Hz}, 2 \mathrm{H}), 1.52(\mathrm{sex}, J=7.3 \mathrm{~Hz}, 2 \mathrm{H}), 0.95$ (t, $J=7.2 \mathrm{~Hz}$, $3 \mathrm{H}) .{ }^{13} \mathrm{C} \mathrm{RMN}: \mathrm{CDCl}_{3}, 100 \mathrm{MHz}, \delta(\mathrm{ppm}): 137.60,129.21,128.19,126.76,123.12,32.72$, 28.94, 22.71, 13.50. IR (KBr, film) v 3064, 2850, 1589, 1493, 955. MS (EI, $70 \mathrm{eV}) \mathrm{m} / z$ (relative intensity): 183 (25), 104 (100), 77 (35). HRMS Calcd. for $\mathrm{C}_{12} \mathrm{H}_{16} \mathrm{Se}: 240.04178$. Found: 240.04252.

4-Butylselanyl-2-methyl-but-3-en-2-ol - 5c: Yield: $0.139 \mathrm{~g}(63 \%) .{ }^{1} \mathrm{H} \mathrm{RMN}: \mathrm{CDCl}_{3}, 200$ MHz, $\delta(\mathrm{ppm}): 6.24(\mathrm{~d}, J=10.3 \mathrm{~Hz}, 1 \mathrm{H}), 6.01(\mathrm{~d}, J=10.3 \mathrm{~Hz}, 1 \mathrm{H}), 2.72(\mathrm{t}, J=7.2 \mathrm{~Hz}, 2 \mathrm{H})$, $2.18(\mathrm{~s}, 1 \mathrm{H}), 1.76$ (quint, $J=7.2 \mathrm{~Hz}, 2 \mathrm{H}), 1.45-1.26(\mathrm{~m}, 8 \mathrm{H}), 0.96(\mathrm{t}, J=7.2 \mathrm{~Hz}, 3 \mathrm{H}) .{ }^{13} \mathrm{C}$ RMN: $\mathrm{CDCl}_{3}, 50 \mathrm{MHz}, \delta(\mathrm{ppm}):$ 137.34, 119.06, 72.46, 32.64, 29.65, 27.71, 23.08, 13.48 . IR $(\mathrm{KBr}$, film) $\vee$ 3289, 3025, 2891, 1709, 1601, 1499, 1213, 949. MS (EI, $70 \mathrm{eV}) \mathrm{m} / z$ (relative intensity): 204 (32), 165 (21), 86 (100), 68 (57). HRMS Calcd. for $\mathrm{C}_{9} \mathrm{H}_{18} \mathrm{OSe}$ : 222.05237. Found: 222.05381.

1-Butylselanyl-3-methyl-pent-1-en-3-ol - 5d: Yield: 0.145 g $(62 \%) .{ }^{1} \mathrm{H}$ RMN: $\mathrm{CDCl}_{3}$, $400 \mathrm{MHz}, \delta(\mathrm{ppm}): 6.26(\mathrm{~d}, J=10.2 \mathrm{~Hz}, 1 \mathrm{H}), 5.91(\mathrm{~d}, J=10.2 \mathrm{~Hz}, 1 \mathrm{H}), 2.62(\mathrm{t}, J=7.2 \mathrm{~Hz}$, $2 \mathrm{H}), 2.04(\mathrm{~s}, 1 \mathrm{H}), 1.68-1.60(\mathrm{~m}, 4 \mathrm{H}), 1.48-1.25(\mathrm{~m}, 2 \mathrm{H}), 1.31(\mathrm{~s}, 3 \mathrm{H}), 0.94(\mathrm{t}, J=7.2 \mathrm{~Hz}$, $6 \mathrm{H}) .{ }^{13} \mathrm{C} \mathrm{RMN}: \mathrm{CDCl}_{3}, 100 \mathrm{MHz}, \delta(\mathrm{ppm}): 137.39,119.62,71.15,35.11,32.68,29.65$, 29.59, 27.23. 13.52, 8.12. IR (KBr, film) v 3361, 3033, 2881, 1658, 1488, 1207, 937. MS (EI, $70 \mathrm{eV}) \mathrm{m} / z$ (relative intensity): 218 (34), 179 (45), 100 (100), 82 (41). HRMS Calcd. for $\mathrm{C}_{10} \mathrm{H}_{20} \mathrm{OSe}: 236.06798$. Found: 236.06843 .

2-Butylselanyl-pent-1-en-3-ol - 5e: Yield: 0.146 g (78\%). ${ }^{1} \mathrm{H}$ RMN: $\mathrm{CDCl}_{3}, 200 \mathrm{MHz}$, $\delta(\mathrm{ppm}): 5.96(\mathrm{~s}, 1 \mathrm{H}), 5.12(\mathrm{~s}, 1 \mathrm{H}), 4.08-4.05(\mathrm{~m}, 1 \mathrm{H}), 2.88-2.51(\mathrm{~m}, 2 \mathrm{H}), 2.3(\mathrm{~s}, 1 \mathrm{H}), 1.79-$ $1.6(\mathrm{~m}, 4), 1.43-160(\mathrm{~m}, 2 \mathrm{H}), 0.96(\mathrm{t}, J=7.2 \mathrm{~Hz}, 6 \mathrm{H}) .{ }^{13} \mathrm{C} \mathrm{RMN} \mathrm{CDCl}_{3}, 50 \mathrm{MHz}, \delta(\mathrm{ppm})$ : $134.50,122.33,72.22,32.69,29.21,26.79,22.95,13.37,9.35 . \mathrm{IR}(\mathrm{KBr}$, film) $v 3382$, 3049, 2865, 1589, 1487, 1219, 929. MS (EI, $70 \mathrm{eV}$ ) m/z (relative intensity): 204 ( 27), 165 (55), 86 (100). HRMS Calcd. for $\mathrm{C}_{9} \mathrm{H}_{18} \mathrm{OSe}$ : 222.05231. Found: 222.05312.

1-(2-Butylselanyl-vinyl)-cyclohexanol - 5f: Yield: $0.156 \mathrm{~g}(60 \%) .{ }^{1} \mathrm{H}$ RMN: $\mathrm{CDCl}_{3}, 400$ $\mathrm{MHz}, \delta(\mathrm{ppm}): 6.24(\mathrm{~d}, J=10.2 \mathrm{~Hz}, 1 \mathrm{H}), 6.01(\mathrm{~d}, J=10.2 \mathrm{~Hz}, 1 \mathrm{H}), 3.08(\mathrm{~s}, 1 \mathrm{H}), 2.62(\mathrm{t}, J=$ $7.2 \mathrm{~Hz}, 2 \mathrm{H}), 1.93-1.89(\mathrm{~m}, 2 \mathrm{H}), 1.69-1.4(\mathrm{~m}, 10 \mathrm{H}), 1.25-1.22(\mathrm{~m}, 2 \mathrm{H}), 0.91$ (t, $J=7.2 \mathrm{~Hz}$, $3 \mathrm{H}) .{ }^{13} \mathrm{C} \mathrm{RMN}: \mathrm{CDCl}_{3}, 100 \mathrm{MHz}, \delta(\mathrm{ppm}): 136.58,119.49,74.83,37.22,32.45,27.56$, 
25.15, 21.84, 21.74, 13.31. IR (KBr, film) v 3391, 3047, 2922, 1637, 1440, 1260, 930. MS (EI, $70 \mathrm{eV}$ ) m/z (relative intensity): 244 (17), 205 (41), 126 (100), 108 (25). HRMS Calcd. for $\mathrm{C}_{12} \mathrm{H}_{22} \mathrm{OSe}$ : 262.08367. Found: 262.08454 .

1-(2-Butylselanyl-vinyl)-piperidine - 5g: Yield: 0.126 g (51\%). ${ }^{1} \mathrm{H}$ RMN: $\mathrm{CDCl}_{3}, 200$ $\mathrm{MHz}, \delta(\mathrm{ppm}): 6.39(\mathrm{dt}, J=9.5 \mathrm{~Hz}, J=1.3 \mathrm{~Hz}, 1 \mathrm{H}), 6.31(\mathrm{dt}, J=9.4 \mathrm{~Hz}, J=4.9 \mathrm{~Hz}, 1 \mathrm{H})$, $2.89(\mathrm{dd}, J=4.9 \mathrm{~Hz}, J=1.2 \mathrm{~Hz}, 2 \mathrm{H}), 2.35-2.29(\mathrm{~m}, 6 \mathrm{H}), 1.66-1.18(\mathrm{~m}, 8 \mathrm{H}), 0.89(\mathrm{t}, J=7.2$ $\mathrm{Hz}, 3 \mathrm{H}) .{ }^{13} \mathrm{C}$ RMN: $\mathrm{CDCl}_{3}, 100 \mathrm{MHz}, \delta(\mathrm{ppm}): 131.24,109.98,63.56,61.40,32.56,23.03$, 22.02, 13.68, 7.02. IR (KBr, film) v 3050, 2961, 2850, 1627, 1449, 951. MS (EI, $70 \mathrm{eV})$ $\mathrm{m} / z$ (relative intensity): 206 (42), 127 (100). HRMS Calcd. for $\mathrm{C}_{11} \mathrm{H}_{21}$ NOSe: 263.07889. Found: 263.07911.

2-Butylselanyl-prop-2-en-1-ol - 6h: Yield: 0.152 g (79\%). ${ }^{1} \mathrm{H} \mathrm{RMN}: \mathrm{CDCl}_{3}, 200 \mathrm{MHz}$, $\delta(\mathrm{ppm}) 5.71(\mathrm{~s}, 1 \mathrm{H}), 5.26(\mathrm{~s}, 1 \mathrm{H}), 4.35(\mathrm{~s}, 2 \mathrm{H}), 2.79(\mathrm{t}, J=7.2 \mathrm{~Hz}, 3 \mathrm{H}), 1.76$ (quint, $J=7.2$ $\mathrm{Hz}, 2 \mathrm{H}), 1.51(\mathrm{sex},=7.2 \mathrm{~Hz}, 2 \mathrm{H}), 0.96(\mathrm{t}, J=7.2 \mathrm{~Hz}, 3 \mathrm{H}) .{ }^{13} \mathrm{C} \mathrm{RMN}: \mathrm{CDCl}_{3}, 50 \mathrm{MHz}$, $\delta(\mathrm{ppm}): 140.98,114.18,66.74,32.71,24.99,22.86,13.38$. IR (KBr, film) v 3377, 3017, 2955, 2863, 1627, 1448, 1179, 968. MS (EI, $70 \mathrm{eV}$ ) $\mathrm{m} / \mathrm{z}$ (relative intensity): 176 (55), 137 (24), 58 (100). HRMS Calcd. for $\mathrm{C}_{7} \mathrm{H}_{14} \mathrm{OSe}$ : 194.02109. Found: 194.02191.

(2-Butylselanyl-vinyl-1-d1)-benzene - 7: Yield $0.192 \mathrm{~g}(80 \%)$; ${ }^{1} \mathrm{H} \mathrm{RMN}: \mathrm{CDCl}_{3}, 200$ MHz, $\delta(\mathrm{ppm}): 7.43-7.21(\mathrm{~m}, 5 \mathrm{H}) ; 6.60(\mathrm{~s}, 1 \mathrm{H}) ; 2.82(\mathrm{t}, J=7.3 \mathrm{~Hz}, 2 \mathrm{H}) ; 1.81-1.38(\mathrm{~m}, 4 \mathrm{H})$, $0.86(\mathrm{t}, J=7.2 \mathrm{~Hz}, 3 \mathrm{H}) .{ }^{13} \mathrm{C} \mathrm{RMN}: \mathrm{CDCl}_{3}, 50 \mathrm{MHz}, \delta(\mathrm{ppm}): 128.62,128.23,126.80$, 123.12, 32.77, 28.65, 22.75, 13.55. IR (KBr, film) v 3050, 3017, 2950, 2921, 2863, 1592, 1582, 1561, 1480, 1452, 1030, 1029, 765, 788. MS (EI, $70 \mathrm{eV}) \mathrm{m} / z$ (relative intensity): 184 (33), 105 (100), 77 (44). HRMS Calcd. for $\mathrm{C}_{12} \mathrm{H}_{15}$ DSe: 241.04795. Found: 241.04852.

2-Butylselanyl-3-phenyl-prop-2-en-1-ol-d - 9: Yield: $0.176 \mathrm{~g}$ (65\%). ${ }^{1} \mathrm{H}$ RMN: $\mathrm{CDCl}_{3}$, $200 \mathrm{MHz}, \delta(\mathrm{ppm}): 7.50-7.22(\mathrm{~m}, 5 \mathrm{H}), 4.45(\mathrm{~s}, 2 \mathrm{H}), 3.06(\mathrm{~s}, 1 \mathrm{H}), 2.78(\mathrm{t}, J=7.2 \mathrm{~Hz}, 2 \mathrm{H})$, $1.59-1.37(\mathrm{~m}, 4 \mathrm{H}), 0.88(\mathrm{t}, J=7.3 \mathrm{~Hz}, 3 \mathrm{H}) .{ }^{13} \mathrm{C} \mathrm{RMN}: \mathrm{CDCl}_{3}, 50 \mathrm{MHz}, \delta(\mathrm{ppm}): 136.57$, $132.43,131.4,128.85,128.10,127.73,127.00,67.40,32.08,24.30,22.58,13.27 . \mathrm{IR}(\mathrm{KBr}$, film) v 3385, 3063, 3021, 2957, 2930, 2875, 1587, 1593, 1572, 1479, 1461, 1033, 1021, 760, 781. MS (EI, $70 \mathrm{eV}$ ) m/z (relative intensity): 253 (41), 214 (37), 135 (100), 117 (18), 77 (14). HRMS Calcd. for $\mathrm{C}_{13} \mathrm{H}_{17}$ DOSe: 271.05858. Found: 271.05923.

Procedure for [2-Butylselanyl-1-(hydroxy-phenyl-methyl)-hex-1-enyl]-phosphonic acid diethyl ester - 11:To a suspension of the elemental selenium $(0.079 \mathrm{~g}, 1 \mathrm{mmol})$ in dry THF $(5 \mathrm{~mL})$ under argon and magnetic stirring was added $n$-butyllithium $(0.4 \mathrm{~mL}$ of a 2.5 $M$ solution in hexane; $1 \mathrm{mmol})$. A yellow solution was formed. Then alkynylphosphonate $(0.286 \mathrm{~g}, 1.2 \mathrm{mmol})$ is added and after $1 \mathrm{~h}$ the benzaldehyde $(0.107 \mathrm{~g}, 1 \mathrm{mmol})$ is added and the solution is stirring at room temperature for $4 \mathrm{~h}$. After this time the mixture is diluted with ethyl acetate $(20 \mathrm{~mL})$ and washed with brine $(2 \times 20 \mathrm{~mL})$. The organic layer is separated, dried $\left(\mathrm{MgSO}_{4}\right)$ and the solvent is evaporated. The residue is purified by silica gel column chromatography eluting with hexane. Yield: $0.369 \mathrm{~g}(78 \%) .{ }^{1} \mathrm{H} \mathrm{RMN}: \mathrm{CDCl}_{3}, 400$ $\mathrm{MHz}, \delta(\mathrm{ppm}): \mathrm{RMN}^{1} \mathrm{H}: \mathrm{CDCl}_{3}, 200 \mathrm{MHz}, \delta(\mathrm{ppm}): 7.44-7.20(\mathrm{~m}, 5 \mathrm{H}), 4.68(\mathrm{~s}, 1 \mathrm{H}), 4.12$ $\left(\mathrm{dq},{ }^{3} J_{\mathrm{PH}}=7.4 \mathrm{~Hz}, J=7.2 \mathrm{~Hz}, 4 \mathrm{H}\right), 2.78(\mathrm{t}, J=7.3 \mathrm{~Hz}, 4 \mathrm{H}), 1.71-1.30(\mathrm{~m}, 14 \mathrm{H}), 0.95(\mathrm{t}, J$ 
$=7.3 \mathrm{~Hz}, 6 \mathrm{H}) \cdot{ }^{13} \mathrm{C}$ RMN: $\mathrm{CDCl}_{3}, 100 \mathrm{MHz}, \delta(\mathrm{ppm}): 157.71\left(\mathrm{~d},{ }_{P-C}^{2}=6.3 \mathrm{~Hz}\right), 142.78$, $128.38,128.03,127.37,126.84,126.81,125.63,110.01\left(\mathrm{~d},{ }^{1} J_{P-C}=188 \mathrm{~Hz}\right), 75.8,61.92(\mathrm{~d}$, $\left.{ }^{2} J_{P_{-C}}=5 \mathrm{~Hz}\right), 36.45,32.48,32.23,30.69,25.64,23.12,22.73,16.33\left(\mathrm{~d},{ }^{3} J_{P_{-C}}=6 \mathrm{~Hz}\right)$, 13.90, 13.48. MS (EI, $70 \mathrm{eV}) \mathrm{m} / \mathrm{z}$ (relative intensity): 444 (45), 405 (21), 326 (100), 238 (18), 191 (75), 173 (62), 82 (35), 77 (39). HRMS Calcd. for $\mathrm{C}_{21} \mathrm{H}_{35} \mathrm{O}_{4} \mathrm{PSe}: 462.14382$. Found: 462.14412.

SELECTED SPECTRA

言
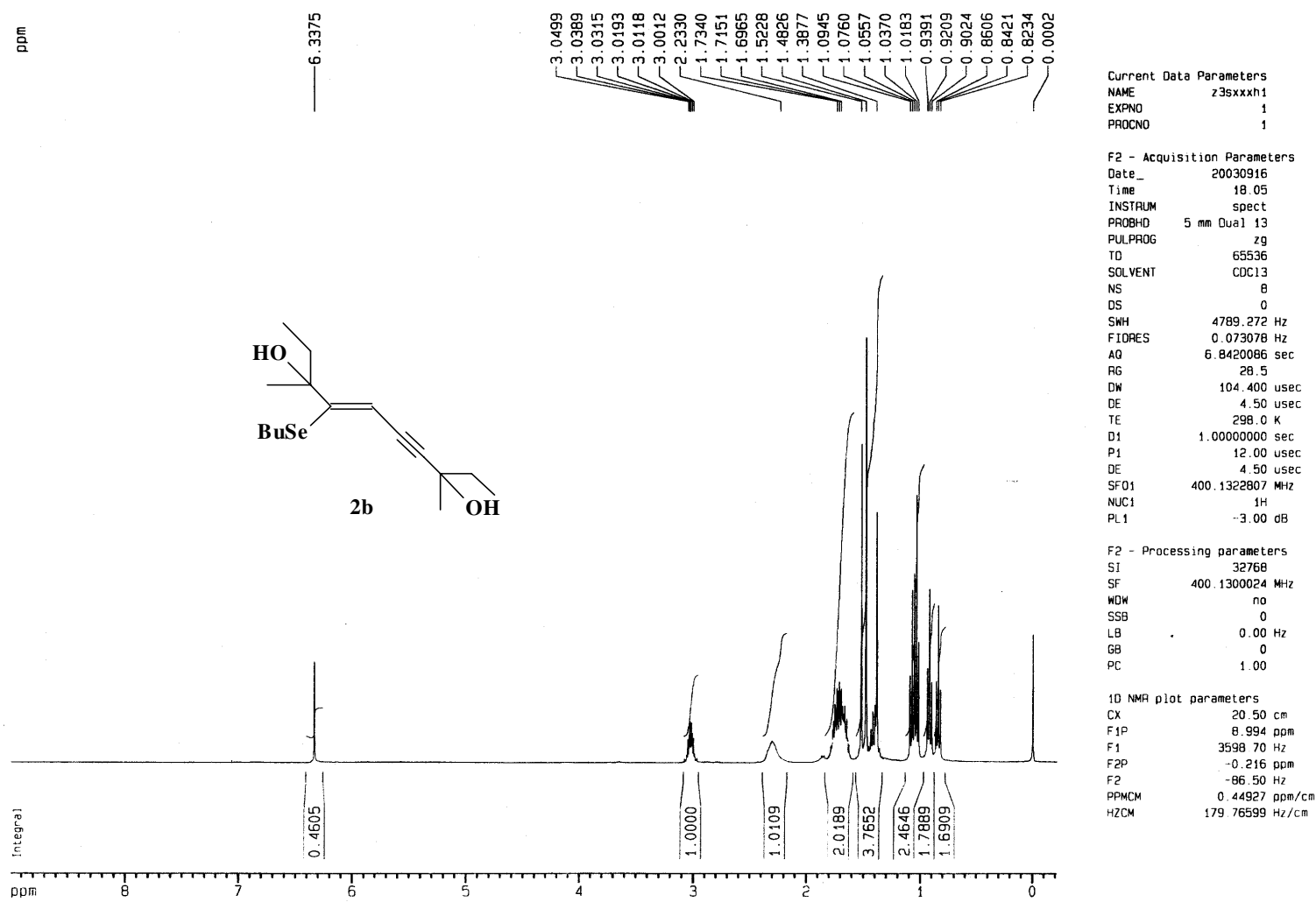


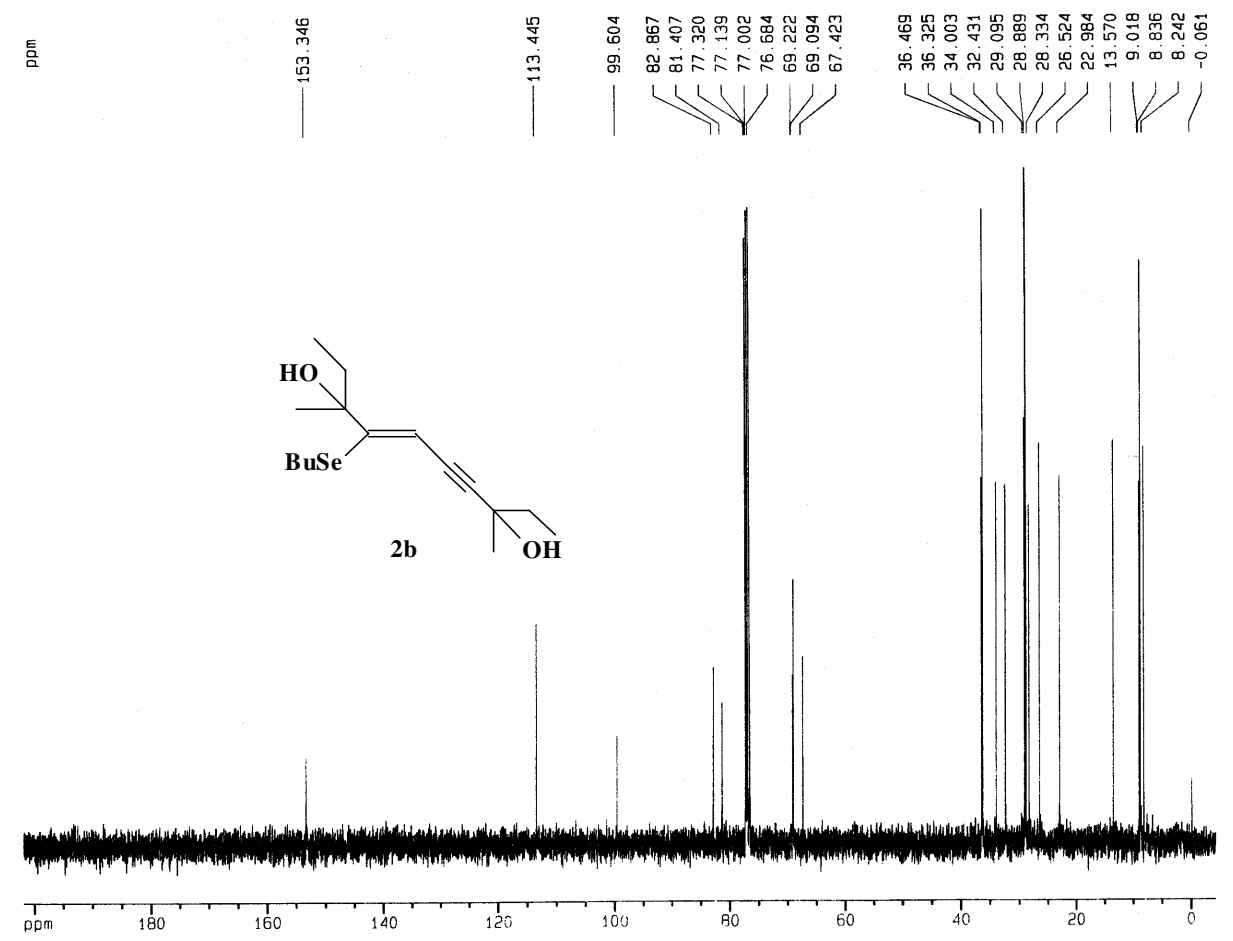

Current Data Parameters
NAME
z3s $3 x \times c c d$

EXPNo

F2 - Acquisition Parameters
Date- 20030916 18.08

PROBHD $5 \mathrm{~mm}$ Dual 13

$\begin{array}{lr}29 \rho 930 \\ \text { PULPHOG } & 65536 \\ \text { COCL13 }\end{array}$

$\begin{array}{lr}\text { SOLVENT } & \text { COCI3 } \\ \text { NS } & 91 \\ \text { DS } & 0\end{array}$

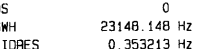

$14156276 \mathrm{sec}$
32768

21.600 usec
4.50 use

$3000 \mathrm{~K}$
000002000

$16.00 \mathrm{~dB}$
$00000000 \mathrm{sec}$

CPDPAG waltz:16

$\begin{array}{ll}106.00 & 115 \mathrm{sec} \\ \mathrm{SFO} & 400.1316005 \mathrm{MHz}\end{array}$

$\begin{array}{ll}12 & -3.00 \mathrm{~dB} \\ 12 & 16.00 \mathrm{~dB}\end{array}$

$7.00 \mathrm{usec}$
$450 \mathrm{usec}$

$1006237964 \mathrm{MH}$

$13 \mathrm{C}$
$-600 \mathrm{~dB}$
$00700000 \mathrm{seC}$

2 - Processing parameters

$\begin{array}{lr}\text { SI } & 32768 \\ \text { SF } & 100.6127721 \mathrm{MHz}\end{array}$

$E M$
SSB
0

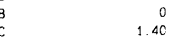

10 NMR plat parameters
CX $21.00 \mathrm{~cm}$
F 19

F! $20314.80 \mathrm{~Hz}$

FPMCM $\quad-42960 \mathrm{~Hz}$

HZCM $\quad 387.8283 ; 47 / \mathrm{C}$

言

푸웜웜

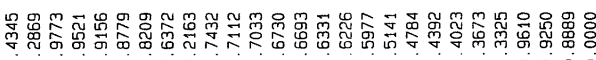

Y

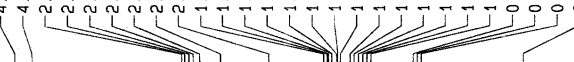

$\begin{array}{lr}\text { NAME } & \text { 230000h1 } \\ \text { EXPND } & 1\end{array}$

F2 - Acquisition Parameters

$\begin{array}{lr}\text { Date } & \\ \text { Time } & 20031006 \\ \text { ines } & 17.09\end{array}$

INSTAMM Spect

$\begin{array}{rr}\text { PAOBHD } & 5 \mathrm{~mm} \text { Dual } \\ \text { PULPAOG } & \text { Z9 }\end{array}$

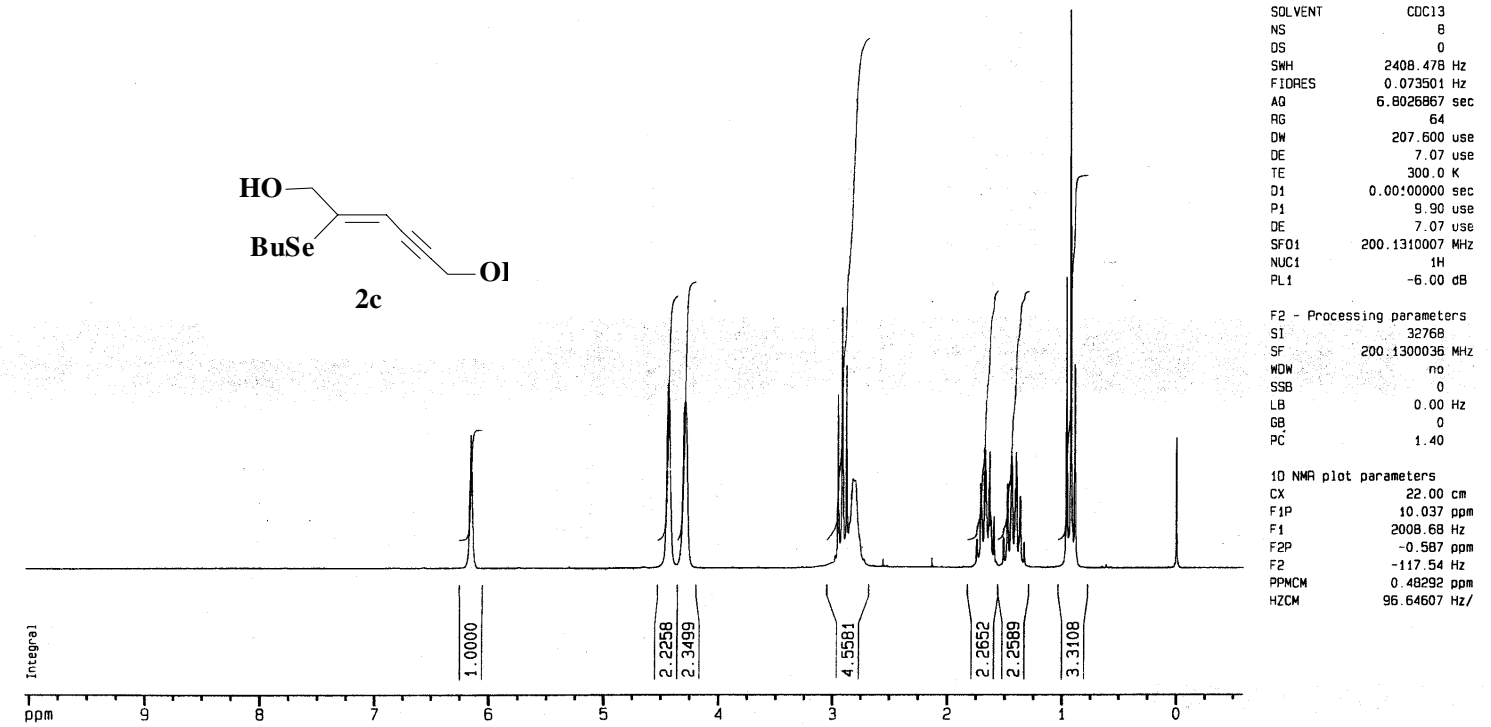


Current Data Parameters
NAME
230000cd

$\begin{array}{lr}\text { EXPNO } & 1 \\ \text { PROCNO } & 2003\end{array}$

F2 - Acquisition Parameters

$\begin{array}{lr}\text { Date } & 20031008 \\ \text { Time } & 15.44\end{array}$

INSTRUM
PROBHD $5 \mathrm{~mm}$ Dual 13

PULPROG $\quad$ 2gpg30

SOLVENT

NS
DS
SWH

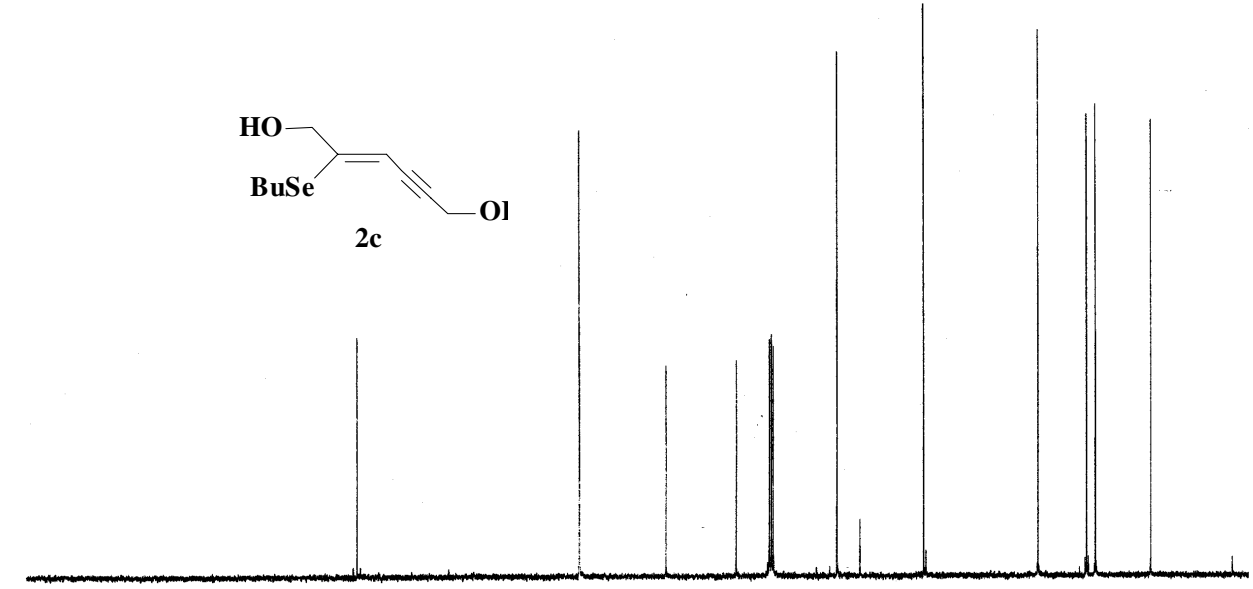

781
0
$23148.148 \mathrm{~Hz}$

IDRES $\quad 0.353213 \mathrm{~Hz}$

$A Q$
AG
B

$\begin{array}{ll}21.600 \text { usec } \\ \text { DE } & 4.50 \text { usec } \\ \text { IE } & 300.0 \mathrm{k}\end{array}$

$0.00002000 \mathrm{sec}$

$\begin{array}{lr}\text { PL13 } & 1600 \mathrm{oB} \\ 01 & 1.20000000 \mathrm{sec}\end{array}$

PPOPAG2 Waltz 16
PCPO2
106

SFO2 400.1316005 MHz

NUC2

$\begin{array}{ll}\text { PL2 } & 300 \mathrm{~dB} \\ \text { PL12 } & 16.00 \mathrm{~dB}\end{array}$

$\begin{array}{ll}P_{1} & 7.60 \mathrm{usec} \\ \mathrm{OE} & 4.50 \mathrm{usec}\end{array}$

SFO1 inO 6237964 MHz

NUC1 - $13 \mathrm{C}$

$\begin{array}{lr}\text { PL1 } & -6.00 \mathrm{~dB} \\ 011 & 0.03000000 \mathrm{sec}\end{array}$

F2 - Processing parameters

$\begin{array}{lr}\text { SI } & 32768 \\ \text { SF } & 100.6127776 \mathrm{MHZ}\end{array}$

$\begin{array}{lc}\text { HOW } & E M \\ \text { SSB } & 0 \\ L B & 1.00 \mathrm{~Hz}\end{array}$

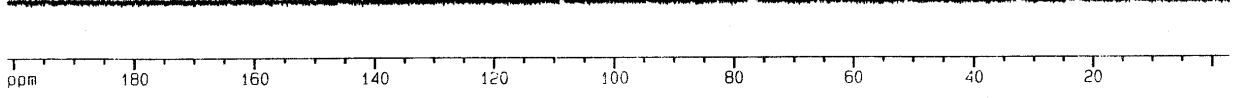

140

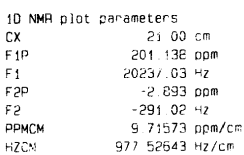

言
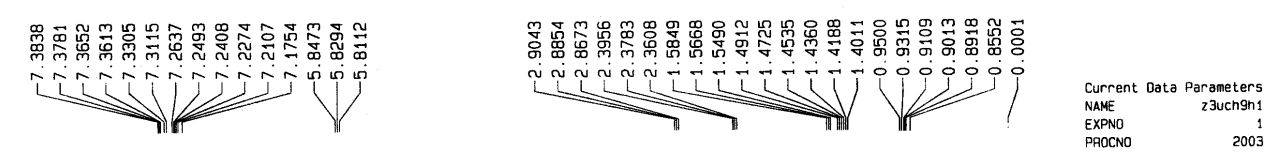

PFOCNO 2003

F2 - Acquisition Parameters

$\begin{array}{lr}20030624 \\ \text { Date } \\ \text { Time } & 18.13 \\ \text { INSTRiM } & \text { spect }\end{array}$

INSTAUM Spect

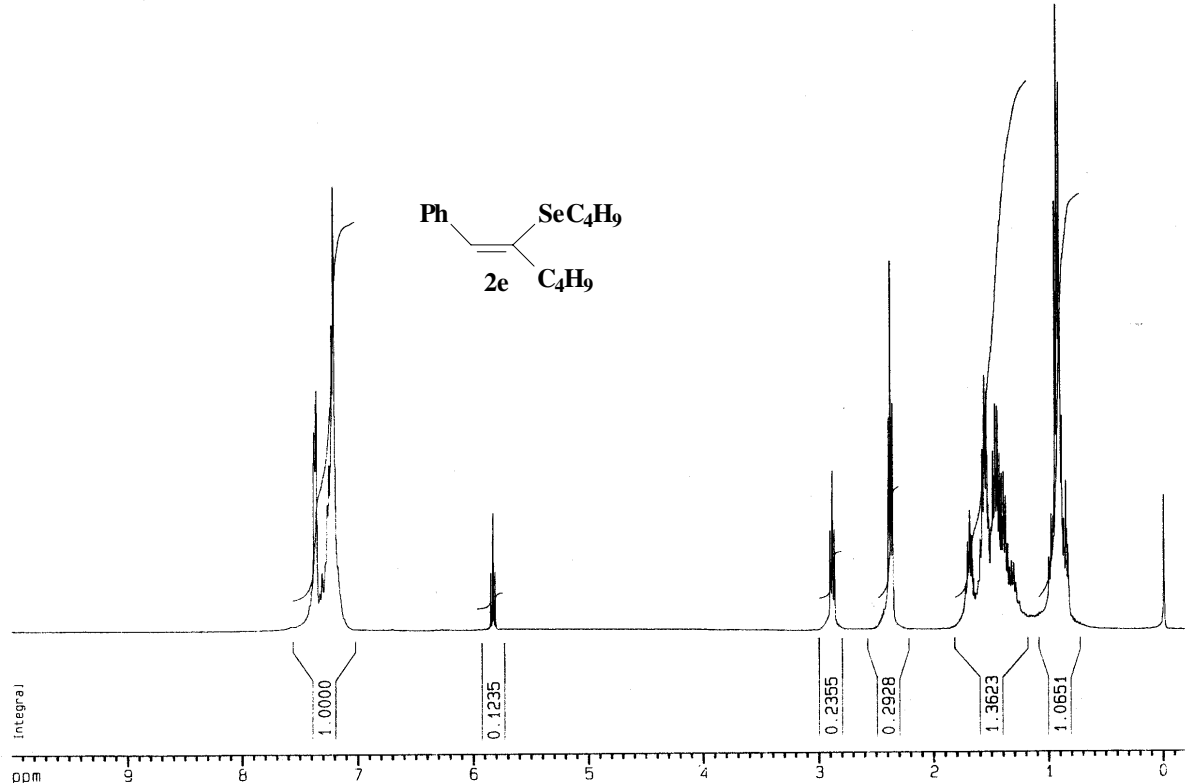

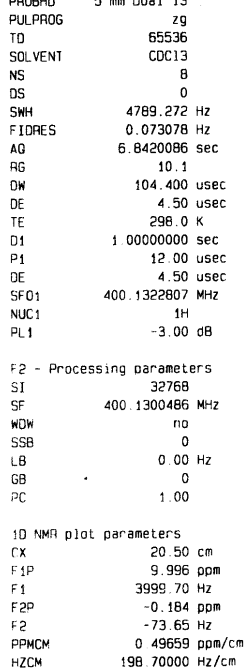
Ppm 


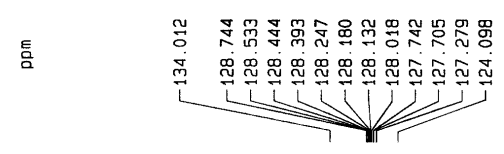

F2 - Acquisition Parameters

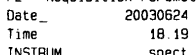

spect
PROBHD $5 \mathrm{~mm}$ Dual

PULPPOG
zgog 30

$\begin{array}{lr}\text { PULPROG } & \text { 290930 } \\ \text { TD } & 65536 \\ \text { SOLVENT } & \text { COC13 } \\ \text { NS } & 115\end{array}$

0
$23148-148$

$\begin{array}{lr}\text { FNH } & 23148.148 \mathrm{~Hz} \\ \text { FIDES } & 0.353213 \mathrm{~Hz} \\ \text { Hat } & 1.4156276 \mathrm{sec}\end{array}$

1.4156276
RG

$\begin{array}{cc}21.600 \text { usec } \\ \text { DE } & 4.50 \text { use } \\ \text { TE } & 3000 \mathrm{~K}\end{array}$

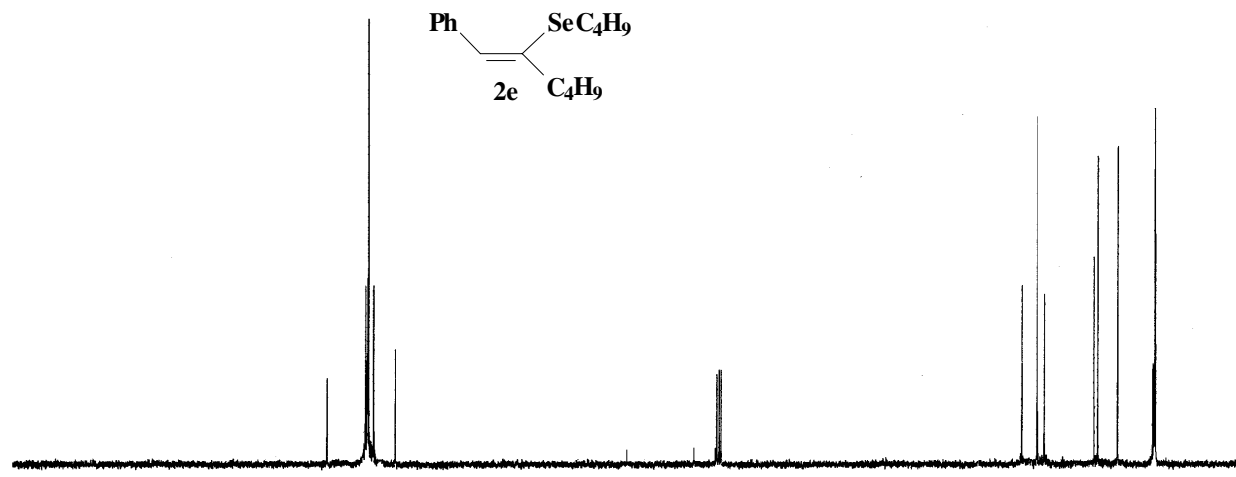

$0.00002000 \mathrm{sec}$

$100000000 \mathrm{de}$

waltz16

$\begin{array}{lr}\text { PCPD2 } & 106.00 \text { use } \\ \text { SF02 } & 400.1316005 \mathrm{MHZ}\end{array}$

$\begin{array}{ll}\text { NUC2 } & 1 \mathrm{H} \\ \text { PLC } & -3.00 \mathrm{~dB}\end{array}$

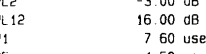

$\begin{array}{lr}\text { DE } & 4.50 \text { use } \\ \text { SF01 } & 100.6237964 \mathrm{MHZ}\end{array}$

NuC1 $\quad 13 \mathrm{C}$

$-6.00 \mathrm{~dB}$
$0.03000000 \mathrm{sec}$

$\begin{array}{lc}\text { F2 - Processing parameters } \\ \text { SI } & 32768 \\ \text { SF } & 100.6127876 \mathrm{MHZ} \\ \text { WOW } & \mathrm{EM} \\ \text { SSB } & 0 \\ \text { B } & 1.00 \mathrm{~Hz} \\ \text { GB } & 0 \\ \text { PC } & 1.40\end{array}$

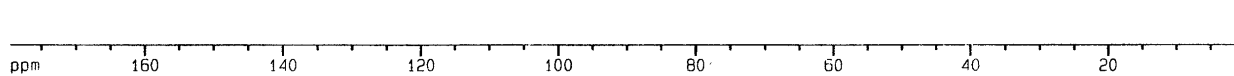

NMF plot parameters
$2100 \mathrm{~cm}$

$\begin{array}{lr}\text { CX } & 21.00 \mathrm{~cm} \\ 51 \mathrm{P} & 179.524 \mathrm{pog}\end{array}$

$\begin{array}{rr}\text { Fip } & 179.524 \mathrm{opm} \\ F & 18062.42 \mathrm{~Hz} \\ +\mathrm{FP} & 1.359 \mathrm{pon}\end{array}$

$\begin{array}{rr}8.88406 \mathrm{ppm} / \mathrm{cm} \\ \text { HZCM } & 353.60443 \mathrm{~Hz} / \mathrm{cm}\end{array}$

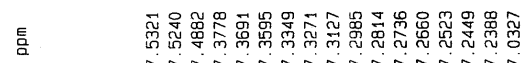

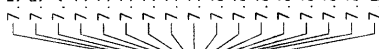

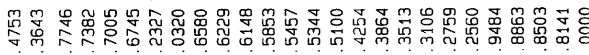
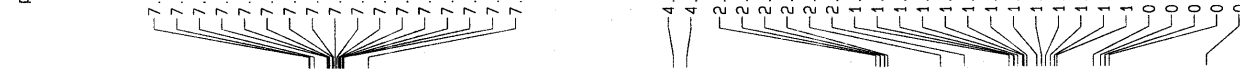

F2 - Acquisition Parameters
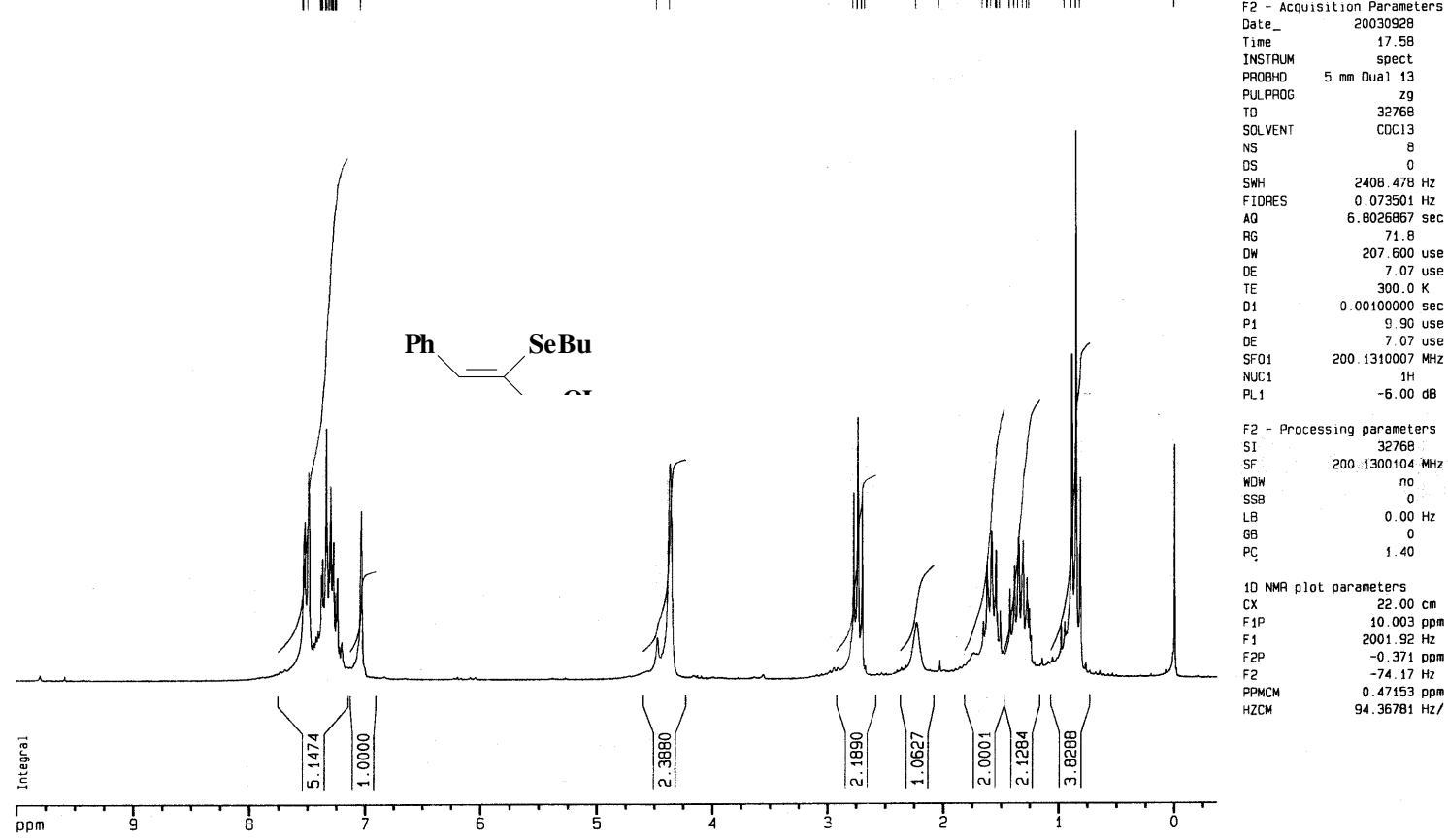


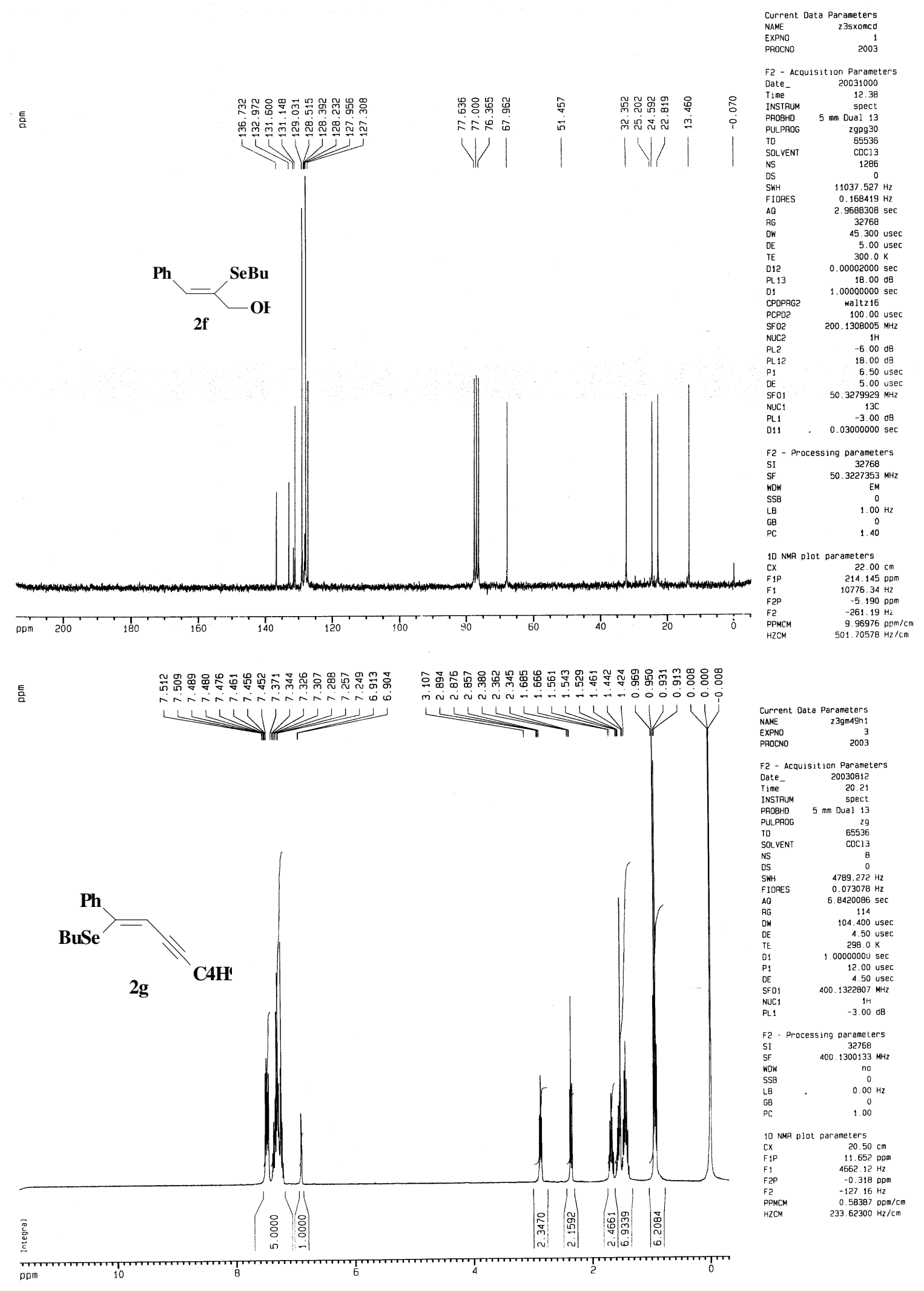



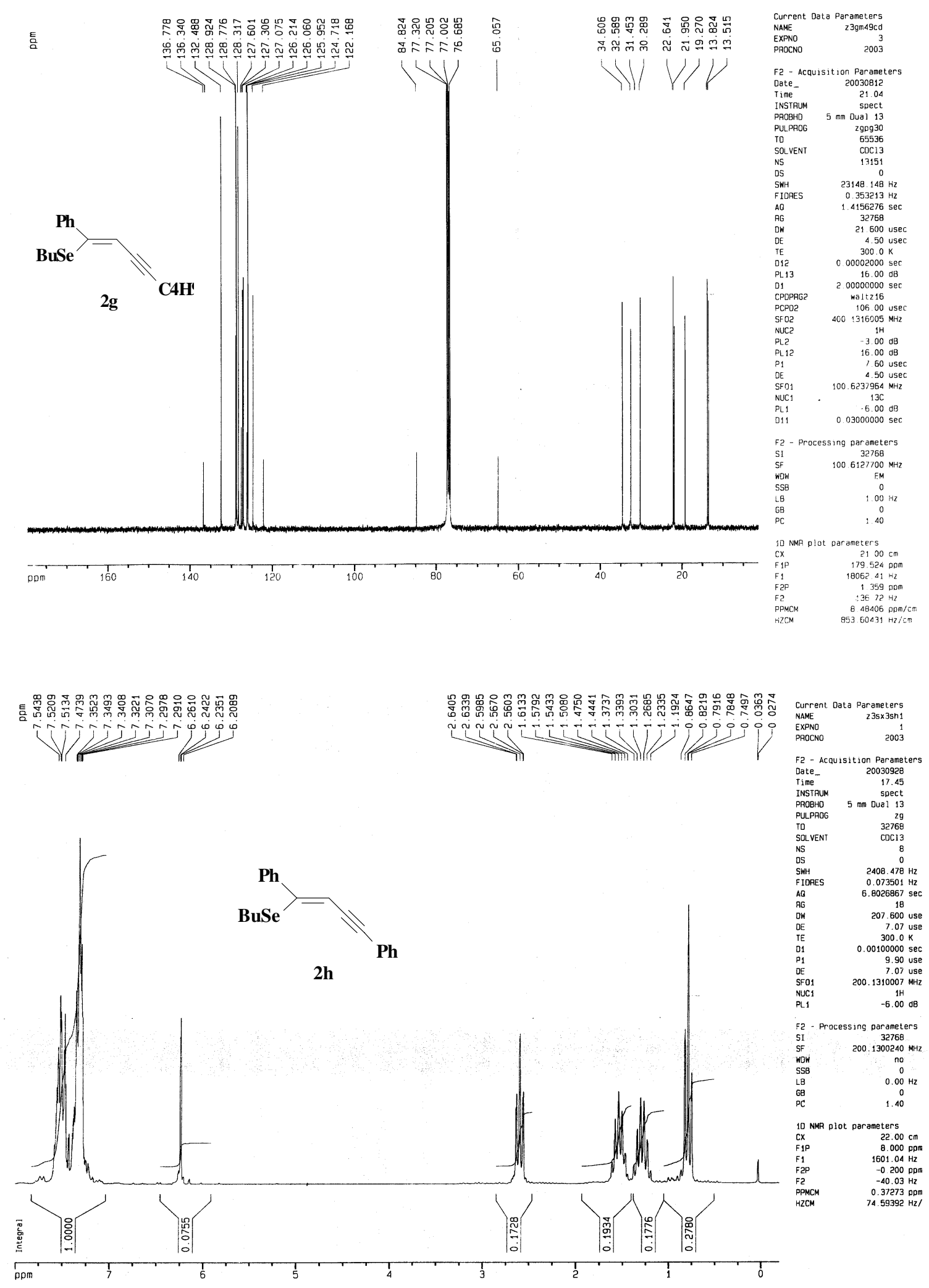

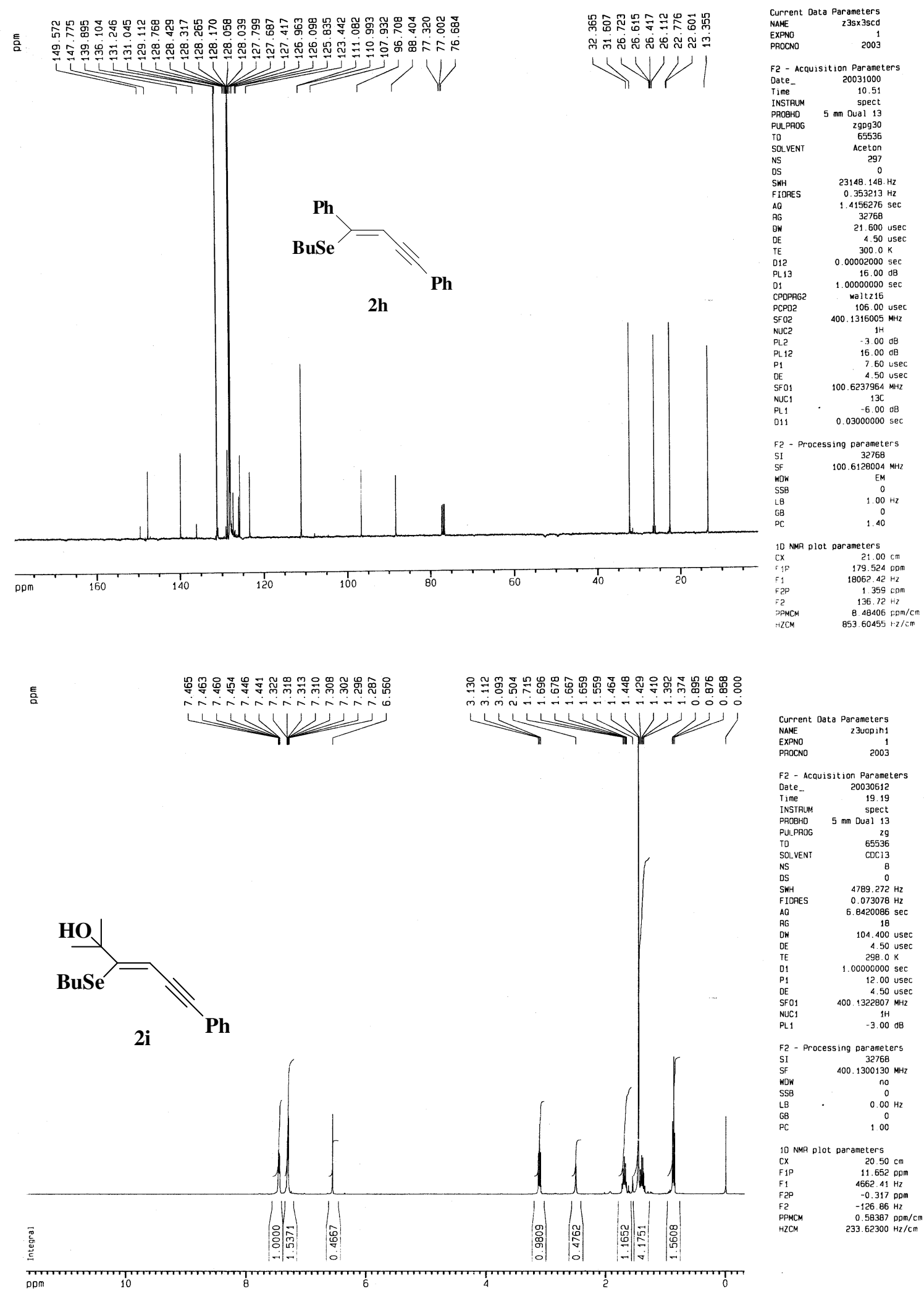


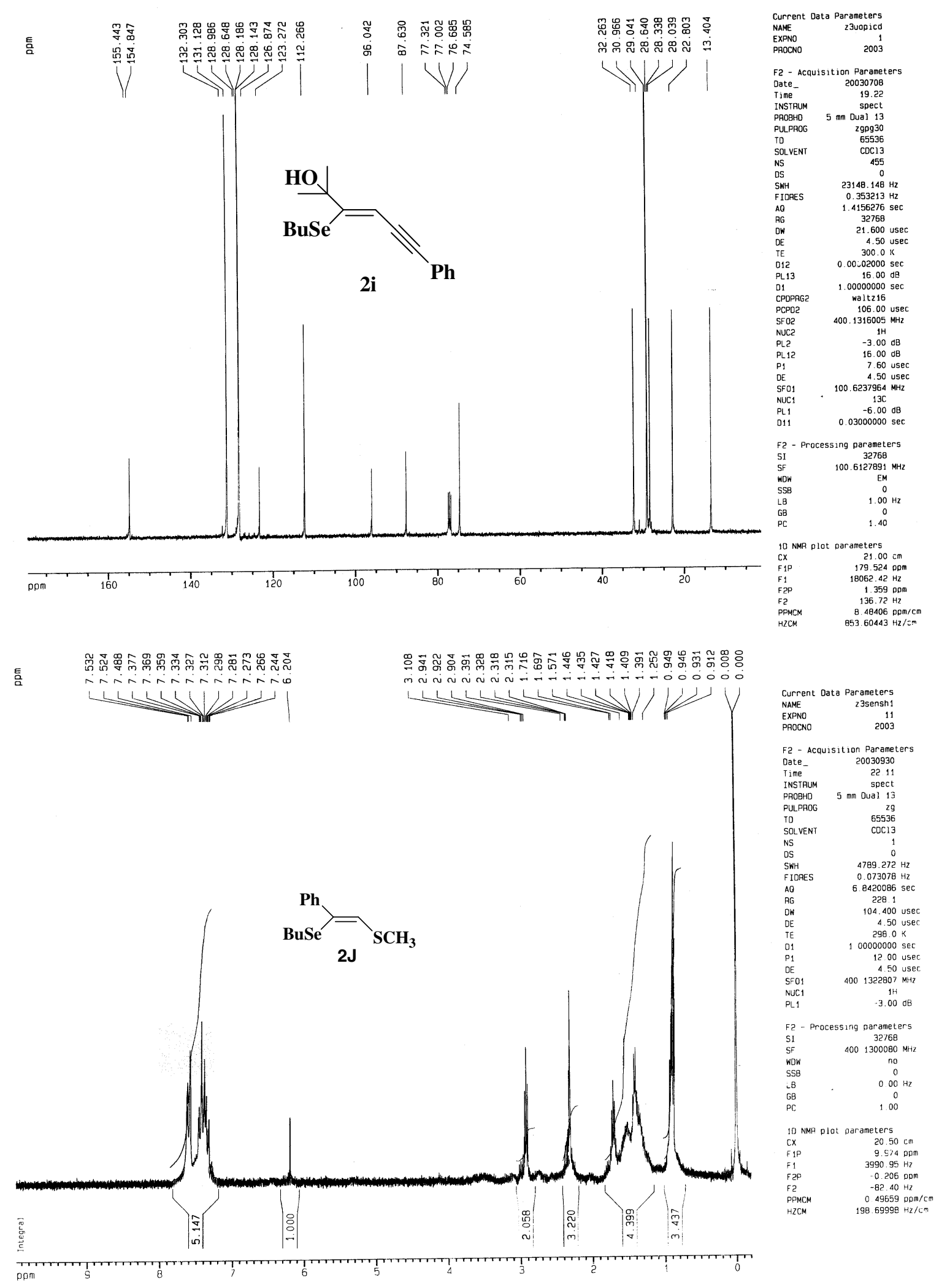



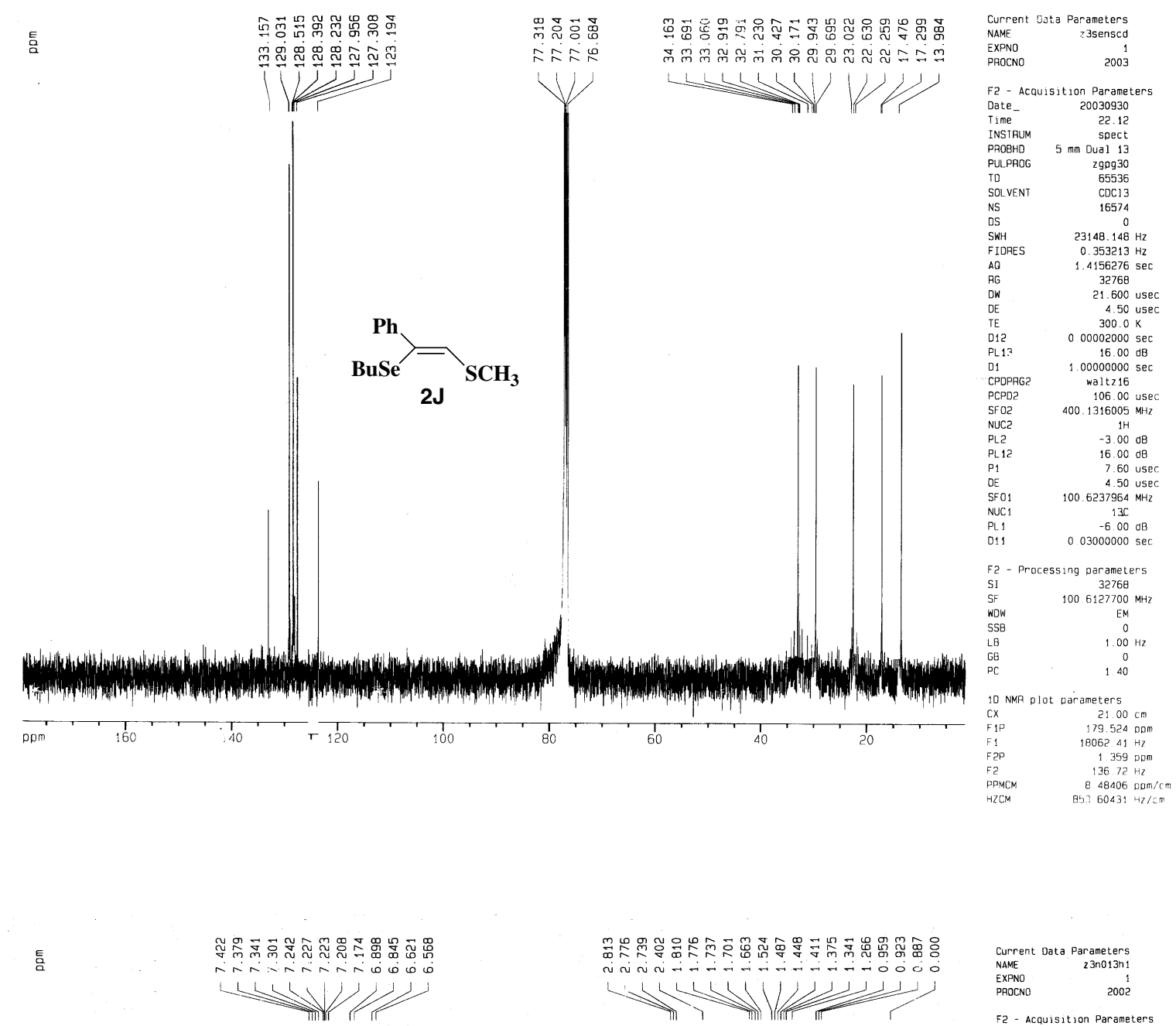

EXPNO
PROCNO 2002
F2- Acquisition Parameters
Date_ 20021122

$\begin{array}{lr}\text { Time } & 16.06 \\ \text { INSTRUM } & \text { spect } \\ \text { PPOBOD } & 5 \mathrm{~mm} \text { Multinu } \\ \text { PULPROG } & 29\end{array}$

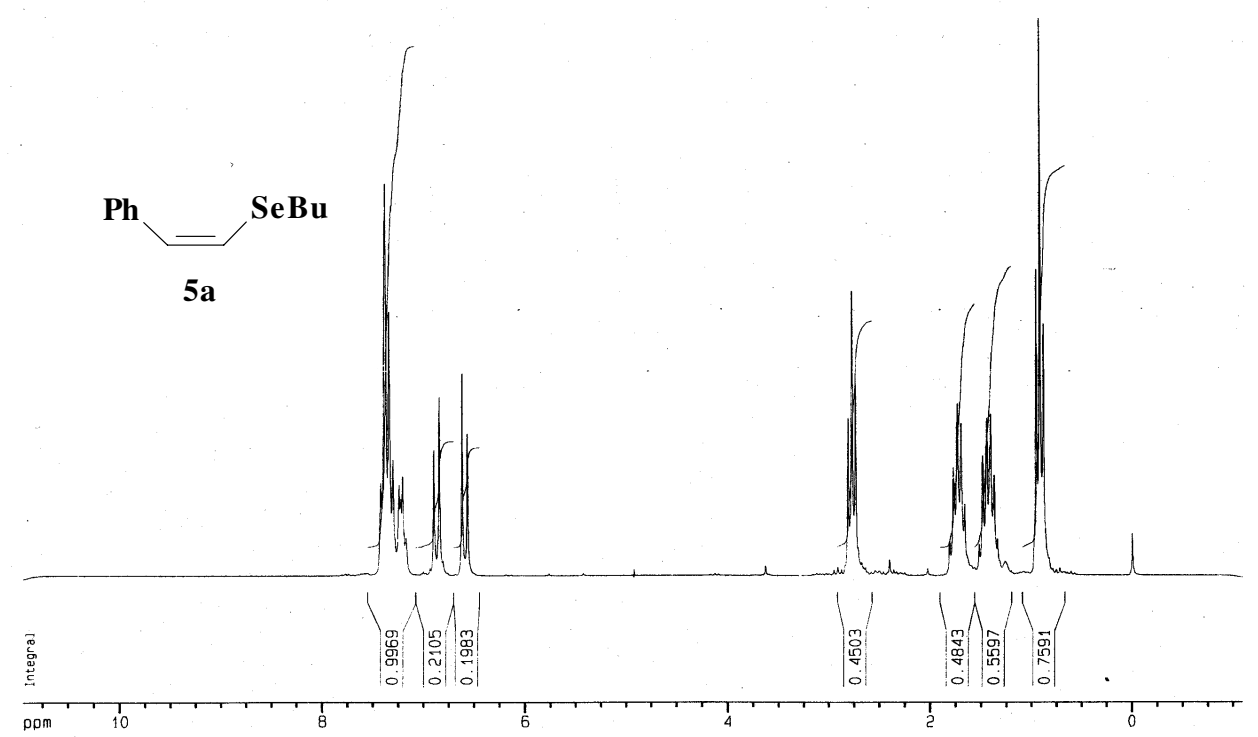

$\begin{array}{lr}29 \\ \text { SOLLENT } & 32768 \\ & \text { COC13 }\end{array}$

$\begin{array}{lc}\text { NS } & \text { CO } \\ \text { DS } & 0 \\ \text { SWH } & 2408.478 \mathrm{~Hz}\end{array}$

$\begin{array}{ll}\text { SWH } & 2408.478 \mathrm{~Hz} \\ \text { FIDAES } & 0.073501 \mathrm{~Hz}\end{array}$

$\begin{array}{ll}\text { AB } & 0.075501 \mathrm{HZ} \\ \mathrm{AB} & 6.8026867 \mathrm{sec}\end{array}$

$\begin{array}{lr}\text { AG } & 57 \\ \text { DW } & 207.600 \text { use } \\ \text { OE } & 7 \text { use }\end{array}$

$\begin{array}{ll}300.0 \mathrm{~K} \\ \mathrm{TE} & 0.00100000 \mathrm{sec} \\ \mathrm{D} & 7.00 \mathrm{se}\end{array}$

7.00 use
7.07 use

SFO1 200.1310007 MHZ

PL1 $-3.00 \mathrm{~dB}$

$\begin{array}{lr}\text { F2 } & \text { Processing parameters } \\ \text { SI } & 32768 \\ \text { SF } & 200.1300153 \mathrm{MHZ}\end{array}$

$\begin{array}{lc}\text { SI } & 32768 \\ \text { SF } & 200.1300153 \mathrm{NHZ} \\ \text { WOW } & \text { no } \\ \text { SSB } & 0 \\ \text { LB } & 0.00 \mathrm{HZ} \\ \text { G8 } & 0\end{array}$

10 NMA plot parameters

$\begin{array}{lr}\text { CX } & 22.00 \mathrm{~cm} \\ \text { F1P } & 10.941\end{array}$

$\begin{array}{ll}F 1 & 2189.59 \mathrm{~Hz} \\ \text { F } & -1.094 \mathrm{pp}\end{array}$

$\begin{array}{ll}\text { F2 } & -218.89 \mathrm{HZ} \\ \text { PPMCM } & 0.54703 \mathrm{pgg}\end{array}$

$\quad 109.47627 \mathrm{~Hz}$ 

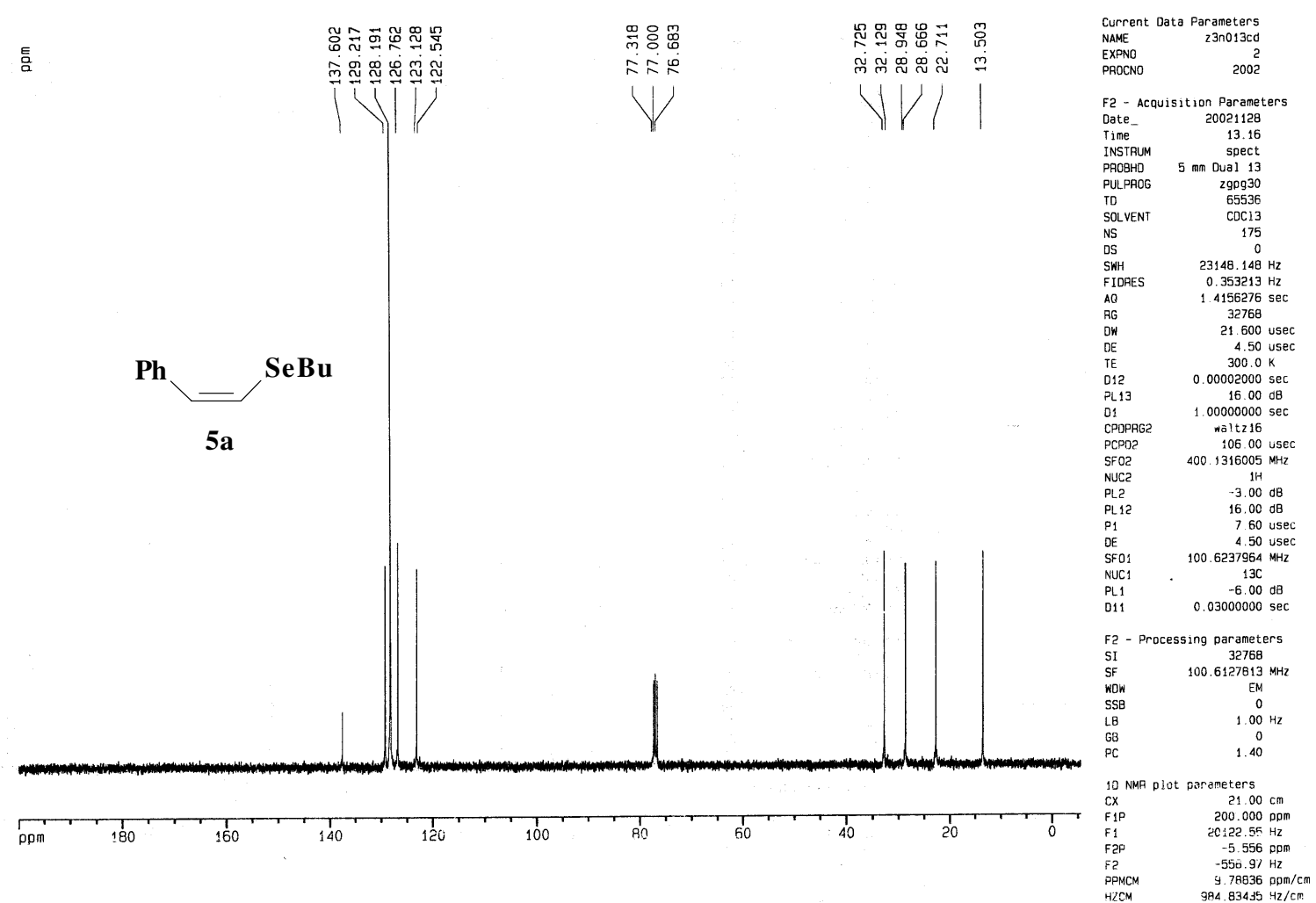

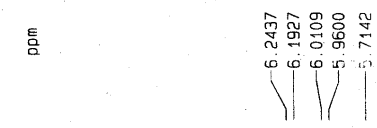

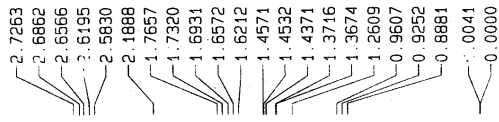

Current Data Parameters
NAME $23,047 \mathrm{~h} 1$

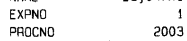

F2- Acquisition Parameters
Date_ 20030124

$\begin{array}{lr}\text { Time r } & 13.31 \\ \text { INSTRUM } & \text { spect } \\ \text { PROBHD } & 5 \mathrm{~mm} \text { Multinu }\end{array}$

PlpaOG

$\begin{array}{lr}\text { PULPROG } & 29 \\ \text { TD } & 32768 \\ \text { SOLVENT } & \text { COC13 }\end{array}$

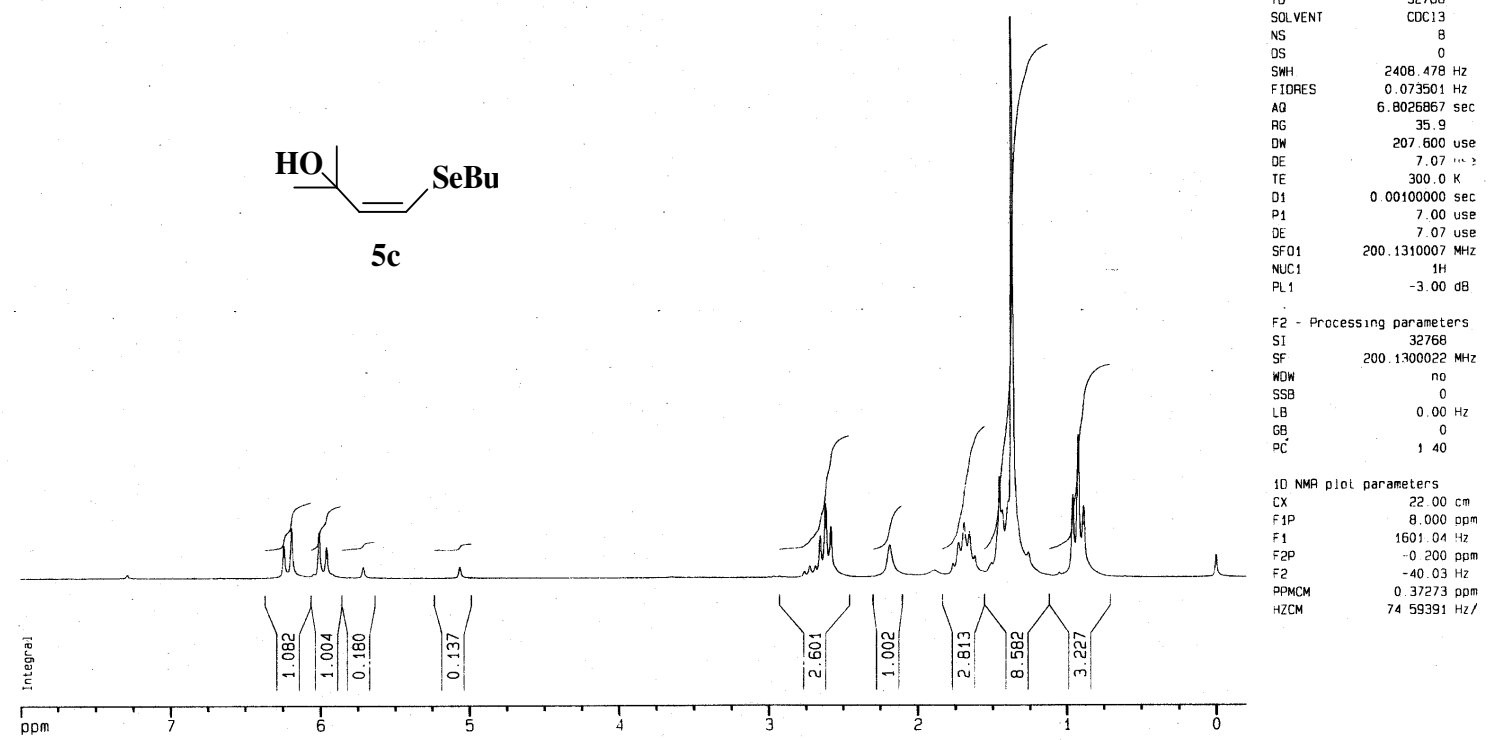




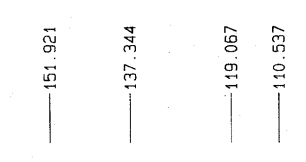

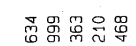

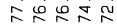

1/1

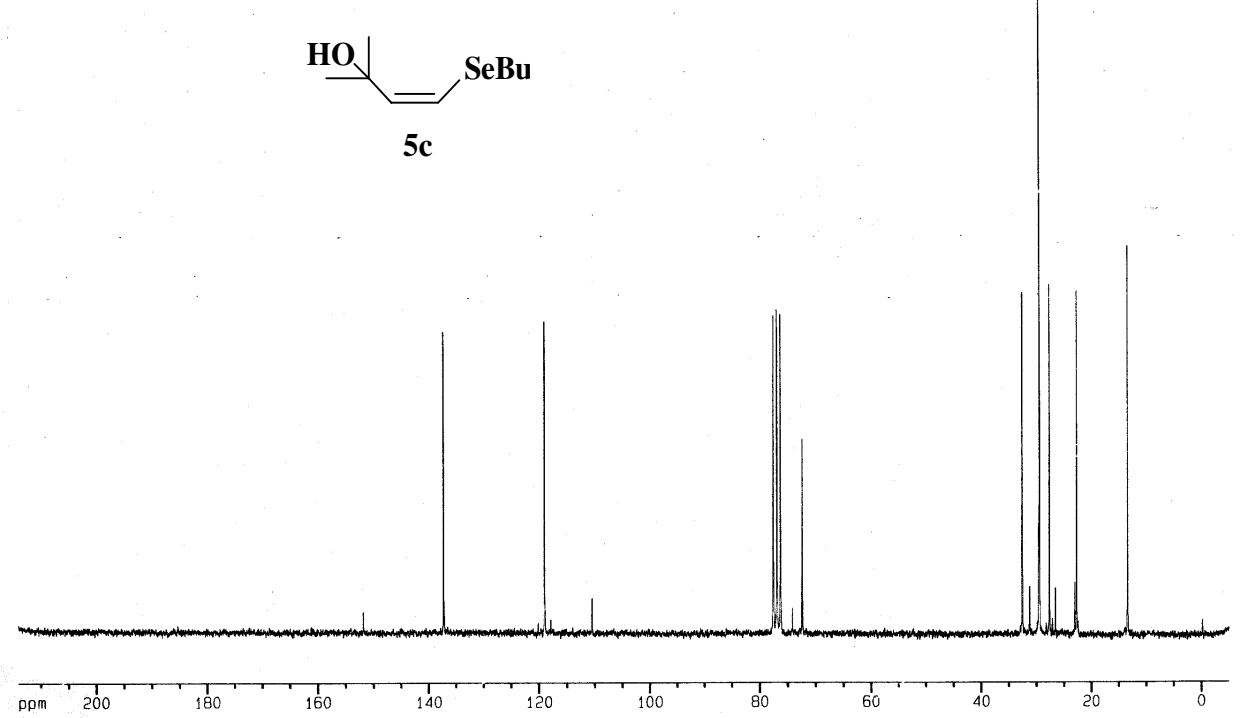

$5 c$

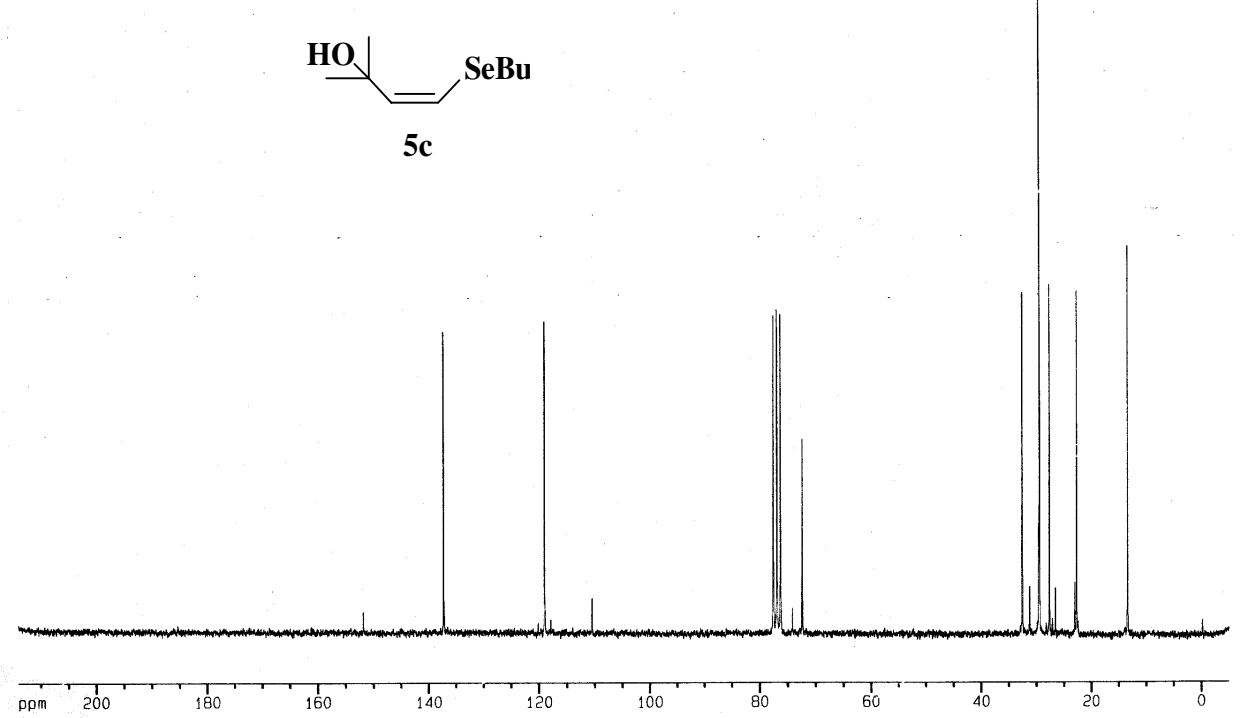

$\overrightarrow{\rho p m}$
Current Data Parameters
NAME $23,047 \mathrm{~cd}$

EXPNO

DROCNO 2003

F2 - Acquisirion Parameters

Date _ $\quad 20030128$

$\begin{array}{lr}\text { Time } & 19.37 \\ \text { INSTRUM } & \text { soect }\end{array}$

PROBHD $5 \mathrm{~mm}$ Multinu

PULPROG 2gpg30

$\begin{array}{ll}\text { TD } & 65536 \\ \text { SOLVENT } & \text { COC13 } \\ \text { NS } & 11427\end{array}$

$\begin{array}{lr}\text { NS } & 11427 \\ \text { DS } & 0 \\ \text { SWH } & 11037527\end{array}$

FIDRES $\quad \begin{array}{r}11037.527 \mathrm{~Hz} \\ 0.168419 \mathrm{~Hz}\end{array}$

AQ $2.9688308 \mathrm{sec}$

DW $\quad \begin{aligned} & 32768 \\ & \text { DW. } 300 \text { usec }\end{aligned}$

TE $\quad 300$ K

$0.00002000 \mathrm{sec}$

$\quad 20.00 \mathrm{~dB}$

$1.00000000 \mathrm{sec}$

waltz16

$110.00 \mathrm{usec}$
$\mathrm{F} 02$

200.1308005
$\mathrm{MHz}$

PL2 $\quad-3.00 \mathrm{~dB}$

$P 1 \quad 11.00$ usec

5.00 usec

50. $3279929 \mathrm{MHz}$

$13 \mathrm{C}$

$-3.00 \mathrm{~dB}$
$11.0 .03000000 \mathrm{sec}$

F2 - Processing parameters

$\begin{array}{lc}\text { SI } & 32768 \\ \text { SF } & 50.3227333 \mathrm{MHz}\end{array}$

$\begin{array}{lr}\text { WOW } & 50.3227333 \\ \text { SSB } & \text { EM }\end{array}$

$\begin{array}{lc}\mathrm{LB} & 1.00 \mathrm{~Hz} \\ \mathrm{~GB} & 0\end{array}$

10 NMA plet parameters
CX $25.00 \mathrm{~cm}$

$\begin{array}{lr}C X & 22.00 \mathrm{~cm} \\ F 1 P & 215.000 \mathrm{ppm}\end{array}$

$\begin{array}{lr}\text { F1 } & 10819.39 \mathrm{~Hz} \\ \text { F2P } & -5.000 \mathrm{ppm}\end{array}$

$\begin{array}{ll}F 2 & -5.000 \mathrm{ppm} \\ F 2 & -251.61 \mathrm{~Hz}\end{array}$

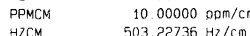

